\title{
Le Clos-Henry : une ferme de La Tène finale à
} Château-Gontier (Mayenne)

Le Clos-Henry, a late La Tene farmstead at Château-Gontier (Mayenne)

Alain Valais, Jean-Claude Meuret, Jean-François Nauleau, Fabrice Edin, Gwenaëlle Hamon et Enzo Muttarelli

\section{OpenEdition}

12 Journals

Édition électronique

URL : https://journals.openedition.org/rao/602

DOI : 10.4000/rao.602

ISBN : 978-2-7535-1608-3

ISSN : $1775-3732$

Éditeur

Presses universitaires de Rennes

Édition imprimée

Date de publication : 20 décembre 2008

Pagination : 139-161

ISBN : 978-2-7535-0789-0

ISSN : 0767-709X

\section{Référence électronique}

Alain Valais, Jean-Claude Meuret, Jean-François Nauleau, Fabrice Edin, Gwenaëlle Hamon et Enzo Muttarelli, «Le Clos-Henry : une ferme de La Tène finale à Château-Gontier (Mayenne) », Revue archéologique de l'Ouest [En ligne], 25 | 2008, mis en ligne le 20 décembre 2010, consulté le 22 août 2022. URL : http://journals.openedition.org/rao/602 ; DOI : https://doi.org/10.4000/rao.602 


\title{
Le Clos-Henry : une ferme de La Tène finale à Château-Gontier (Mayenne)
}

\author{
Le Clos-Henry, a late La Tene farmstead at Château-Gontier (Mayenne)
}

\author{
Alain Valais*, Jean-Claude Meuret** et Jean-François Nauleau*** \\ avec la collaboration de Fabrice Edin****, Gwenaëlle Hamon ${ }^{* * * * *}$ \\ et Enzo Muttarelli ${ }^{* * * * * *}$
}

\begin{abstract}
Résumé : La fouille du site du Clos-Henry à Château-Gontier, outre la découverte d'une fosse du néolithique ancien VSG a permis de mettre en évidence une ferme de La Tène finale d'emprise très modeste. Son enclos rectangulaire à partition interne n'occupe en effet que $2400 \mathrm{~m}^{2}$. Malgré un nombre restreint de structures en creux, moins de quarante trous de poteau, quatre bâtiments sur poteaux y ont été relevés. L'occupation relativement courte du site, une ou deux générations, permet d'y distinguer une unité agricole constituée d'un bâtiment principal associé à quelques bâtiments annexes de type grenier. L’ensemble est établi au cours de la seconde moitié du second siècle avant J.-C. et abandonné au milieu du siècle suivant.
\end{abstract}

\begin{abstract}
The Clos-Henry excavation at Château-Gontier (Mayenne) revealed - in addition to a "VSG" early Neolithic pit - a modest late La Tene farmstead, a rectangular enclosure with internal partition $2400 \mathrm{sq} . \mathrm{m}$. in area. In spite of the restricted number of features identified (less than 40 postholes), four timber-framed buildings have been distinguished. The relatively short occupation of the site (one or two generations) visualises an agricultural unit consisting of a main building associated with some granary-type annexes. The whole is established during the second half of $2^{\text {nd }}$ century $B C$ and abandonned by the middle of the following century.
\end{abstract}

Mots clés : Céramique, VSG, Tène finale, enclos à partition interne, bâtiments.

Key words: Ceramics, VSG Neolithic, late La Tene, partitioned enclosure, buildings.

\section{Présentation}

\section{Localisation du gisement et environnement naturel}

Le site archéologique découvert à proximité de la ferme du Clos-Henry est implanté à la périphérie sud-ouest de Château-Gontier (fig. 1), sur les rebords sud-est d'un pla- teau entaillé par la vallée d'un modeste ru (fig. 2). À quelques centaines de mètres plus à l'est, celui-ci se jette dans le ruisseau du Ponceau, cours d'eau qui vient ensuite grossir les eaux de la Mayenne.

Les vestiges sont établis à $50 \mathrm{~m}$ d'altitude, entre la colline de la Pinaudière $(83 \mathrm{~m})$ à l'ouest et celle d'Yvano $(84 \mathrm{~m})$ au sud. Le substrat se compose principalement de limons issus

* INRAP, 4 rue du Tertre, 44477 Carquefou. (alain.valais@inrap.fr)

** Maître de conférences en archéologie nationale honoraire, La Bitière, 35640 Chelun.

*** INRAP, 4 rue du Tertre, 44477 Carquefou.

**** INRAP, 37 rue du Bignon, 35577 Cesson-Sévigné.

***** Kérispern, 56550 Belz.

***** INRAP Haute-Normandie, 22 rue Nétien, 76000 Rouen. 


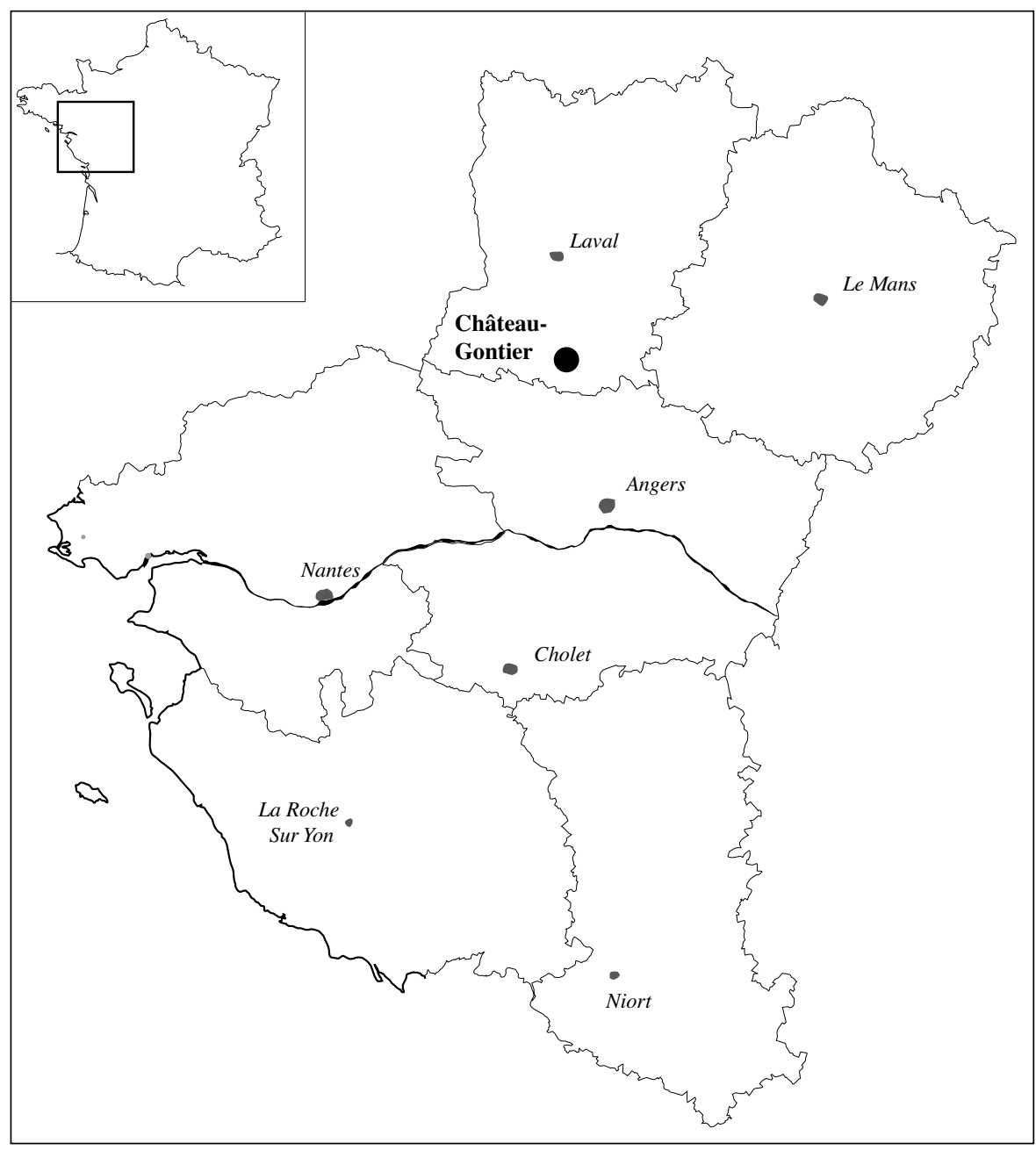

Figure 1 : Localisation générale (sauf précision toutes les illustrations de cet article ont été réalisées par J.-F. Nauleau).

Figure 1: General location map (unless specified, illustrations of this paper are by J.-F. Nauleau). de l'altération des schistes précambriens. Le tout est recouvert par un horizon humique d'une trentaine de centimètres. Les phases détritiques ont une puissance variable avec des minima atteints à l'est du décapage, où le substrat rocheux apparait seulement sous $0,10 \mathrm{~m}$ de limons.

\section{Contexte archéologique}

Dans la région de Château-Gontier, les premiers indices archéologiques remontent à la fin du Néolithique. Ils se résument à un dolmen à la Pêcherie (fig. $2, \mathrm{n}^{\circ} 1$ ) et à un polissoir découvert en 1896 à la Chiffanerie d'Azé ( $\left.{ }^{\circ} 2\right)$ (Angot, 1900, tome 1, p. 660 b).

Il faut attendre la fin de La Tène pour connaître l'existence de populations dans le bassin de Château-Gontier. L'oppidum de la Petite Frizelière ou de la Cadurie, situé aux confins des communes de Château-Gontier/Bazouges et Loigné (fig. 2, $n^{\circ} 3$ ), constitue un éperon barré d'une douzaine d'hectares. Cette enceinte est défendue au nord par un rempart très érodé, et par des falaises de plus de $40 \mathrm{~m}$ au sud-ouest et au sud-est. Elles dominent la confluence du ruisseau du Bouillon et de la Mayenne. Même si la datation de cette enceinte reste incertaine, sa position sur la Mayenne évoque les autres exemples connus dans cette vallée encaissée que sont les oppida d'Entrammes et de Moulay, deux exemples très majoritairement occupés durant La Tène finale (Valais, 2004 et 2008). Pour l'éperon barré de la Petite Frizelière, on évoque au XIX ${ }^{e}$ siècle de très nombreux murs aux Morillands (Angot, 1910, t. 3, p. 123) ou à la Cité, une ferme encore mentionnée sur le cadastre napoléonien, ainsi que des découvertes monétaires gauloises comme un statère d'or attribué aux Cénomans (type BN 6852) (Naveau, 1992, p. 87) et gallo-romaines. Ces éléments épars paraissent confirmer une occupation des lieux depuis La Tène finale jusqu'à la période romaine.

Au sud de Château-Gontier, les sites des Fosses (ex-commune de Bazouges) (fig. 2, $\mathrm{n}^{\circ}$ 5), et un peu plus loin de La Réhaudière sur la commune de Saint-Fort (en dehors de la carte), constituent les traces les plus orientales d'une exploitation aurifère protohistorique et antique que l'on 


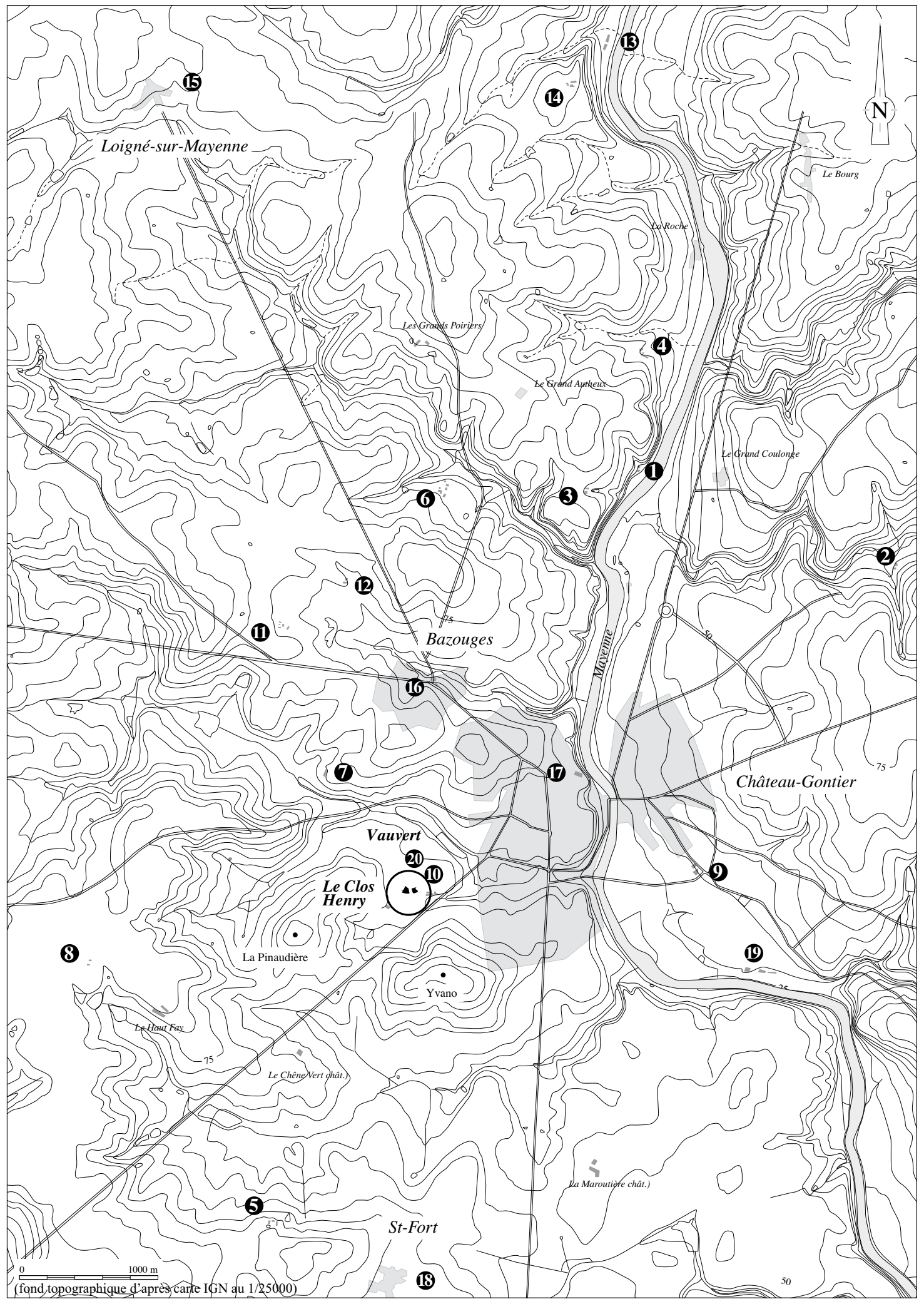

Figure 2 : Contexte archéologique de la région de Château-Gontier (fond topographique d'après carte IGN au $1 / 25000)$; les numéros de sites renvoient au texte

Figure 2: Archaeological context of Château-Gontier area (on French IGN survey topographical background); site numbers refer to text 
suit vers l'ouest jusqu'à La Selle Guerchaise (Ille-et-Vilaine) (Meuret, 1993, p. 231). La présence de cette ressource minière pourrait en partie expliquer la profusion d'enclos que les missions de photographie aérienne nous dévoilent tout au long du " chemin des Miaules" (ibid., p. 203-205). La région de Château-Gontier montre également de nombreux indices d'occupation, que les campagnes aériennes de G. Leroux permettent d'entrevoir. Des sites enclos ont été relevés par exemple à la Bouteillerie (fig. $2, \mathrm{n}^{\circ} 6$ ), à la Guesnonière $\left(\mathrm{n}^{\circ} 7\right)$, à Richebourg $\left(\mathrm{n}^{\circ} 8\right)$, tous repérés sur l'ancienne commune de Bazouges. À ce jour, seul le site de la Mazure $\left(\mathrm{n}^{\circ} 9\right)$, repéré du ciel en 1974 par C. Lambert et J. Rioufreyt et menacé par la construction de la rocade sud de Château-Gontier, a fait l'objet de trop rapides sondages archéologiques l'année suivante (Lambert et Rioufreyt, 1976). Le site, en rive gauche de la Mayenne, se compose d'un triple enclos de forme trapézoïdale; il a essentiellement livré des indices d'occupation du second siècle avant J.-C. (céramique et fragments de plaques foyères). L'existence d'un habitat antique à proximité est étayée par au moins un fossé recelant du mobilier du second siècle après J.-C. (ibid., p 31). L'hypothèse d'une voie romaine peut être proposée ici. Il pourrait s'agir du tracé signalé comme antique et qui longe la rive gauche de la Mayenne depuis Entrammes et Villiers-Charlemagne au nord (Naveau, 1997, fig. 18). Au sud de la paroisse de Fromentière, elle franchirait le ruisseau du Pomperdreau au niveau de la ferme du Petit Moulin pour ensuite gagner Château-Gontier en empruntant l'actuelle rue Trouvée puis celle des Martyrs en limite de zones inondables. Au-delà, l'axe longerait le côté ouest de l'enceinte gauloise de la Mazure pour se diriger au sud vers Azé, siège d'un monastère mentionné entre 529 et 550 (Legros, 2006, p. 35).

La découverte en 2001 de plusieurs indices de sites de l'âge du Fer sur le tracé de la rocade nord de ChâteauGontier/Bazouges au nord des fermes de la Morlière (fig. 2, $\left.n^{\circ} 11\right)$ et de la Petite Jariais ( $\left.{ }^{\circ} 12\right)$ ou encore au lieu-dit Montaigu dans le même secteur (Valais, 2001a), confirme encore l'importance de l'occupation gauloise du secteur (Langlois, 2001).

\section{Circonstance de la découverte}

La découverte du site du Clos Henry est intervenue fortuitement dans une zone pavillonnaire au cours de la viabilisation. Deux fossés perpendiculaires intéressants furent alors identifiés en raison de leurs orientations différentes de celles du parcellaire environnant (fig. 3). À la suite d'un sondage et de l'exploration rapide des coupes, du mobilier (céramique et fragments de terre cuite) fut recueilli permettant d'attribuer l'ensemble des vestiges à La Tène finale. Les relevés et les quelques photographies réalisés ont permis de geler temporairement les aménagements de cette partie du projet avant qu'une opération archéologique ne vienne évaluer l'importance des vestiges mis au jour (Valais, 2001c). Notons encore que ces éléments ont été découverts à $150 \mathrm{~m}$ environ du site haut Moyen Âge de Vauvert, repéré du ciel par G. Leroux en 1992 et qui a fait l'objet d'une évaluation en 1999 (Meuret, 2003, p. 25-30) et d'une fouille en en 2001 (Valais, 2001b) (fig. 2, n² 20).

\section{Une fosse VSG}

Au cours de cette opération, une fosse de forme oblongue (F 40) a été relevée au sud-est de l'enclos de La Tène (fig. 3). Elle mesure $3 \mathrm{~m}$ sur $0,50 \mathrm{~m}$ et est conservée sur $0,20 \mathrm{~m}$ de profondeur. En plus d'un polissoir ou aiguisoir en schiste ou gneiss poli, ce creusement a livré un très intéressant lot céramique constitué de 127 fragments (fig. 4). Parmi les formes identifiées, quatre récipients en trois-quarts de sphère présentent dans leur partie haute un registre décoratif de boutons d'environ $2 \mathrm{~cm}$ de diamètre et des décors de cordons en $V$ à la base desquels sont implantées des anses à perforation horizontale. Ces compositions décoratives commencent à mieux être connues pour le Néolithique ancien (groupe de Villeneuve-Saint-Germain), que ce soit en Basse-Normandie comme à Valframbert ou à Mondeville (Chancerel et al., 1995), au Haut-Mée (Cassen et al., 1998), site fouillé plus récemment en Ille-et-Vilaine, ou dans le Maine-et-Loire à la Bajoulière (Gruet, 1986).

Le décapage réalisé autour de cette fosse n’a révélé aucune autre structure intéressante. L'ensemble a été daté par radiocarbone aux alentours de 5000 avant J.-C. (Lyon 2054 : $6115 \pm 60 \mathrm{BP}$, soit 5255 à $4815 \mathrm{BC}$ - dates les plus probables par ordre décroissant : 5040, 5006, 5185-4965 avant J.-C.).

\section{Un ENSEMble de FOSSÉS de LA TÈnE FINALE}

\section{Un enclos}

D'abord repéré dans sa partie nord-est et ponctuellement dans des tranchées de réseau sur son côté sud-est, l'enclos a finalement été perçu dans sa totalité lors de l'opération d'évaluation du printemps 2001 (fig. 5). L'enceinte, orientée nord-ouest/sud-est, mesure $40 \mathrm{~m}$ de largeur pour une soixantaine de mètres de longueur. Sa surface atteint ainsi $2400 \mathrm{~m}^{2}$ et ses limites ont pu être relevées en continu sur trois côtés (fossé F 7).

Le côté sud-est de l'enclos a été vu lors des travaux de viabilisation, sans que son profil précis ait pu être partout 


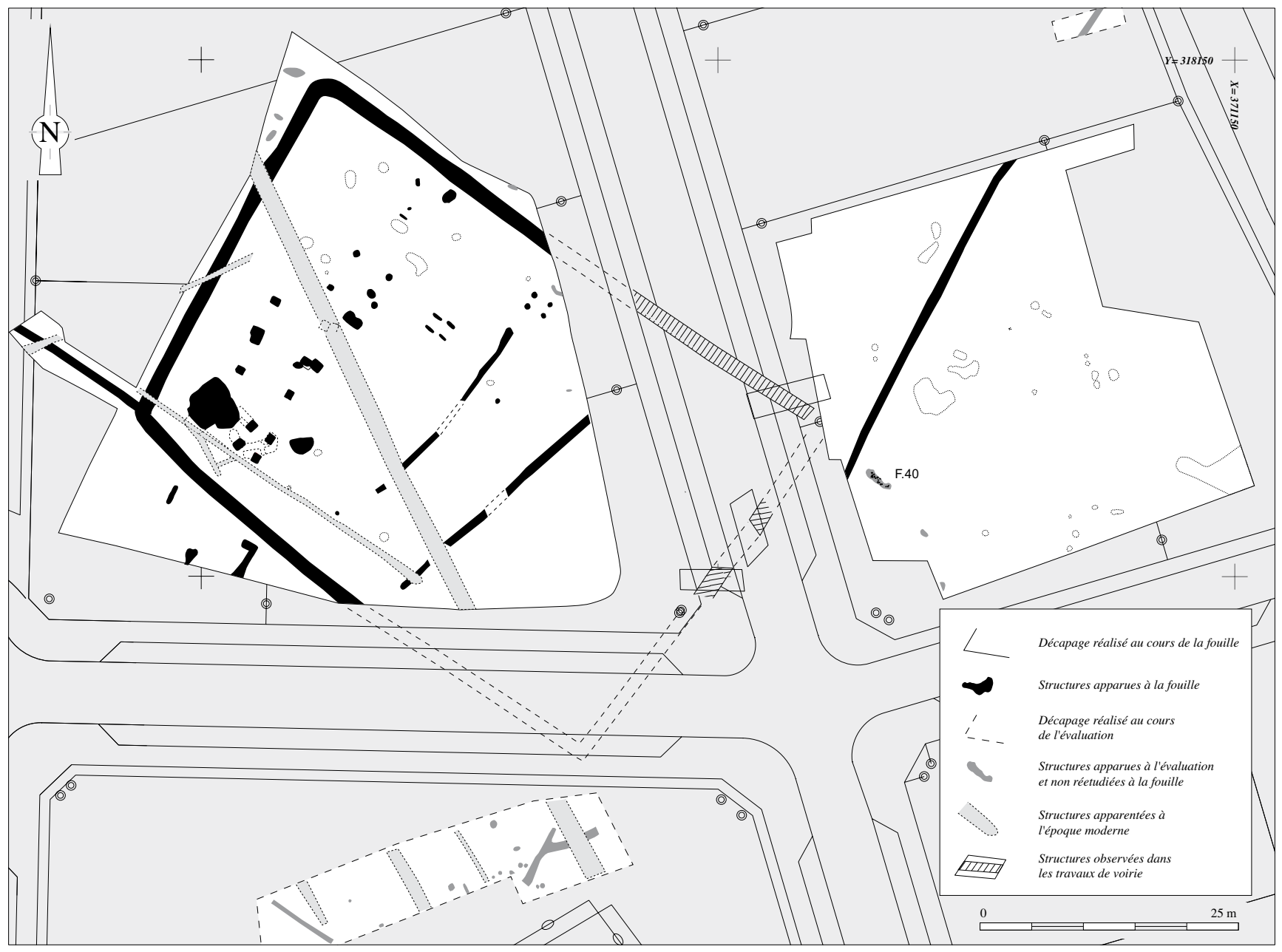

Figure 3 : Plan général de la fouille

Figure 3: General plan of the excavation.

relevé. Les autres fossés ont pu être sondés à la main au cours de l'évaluation et de la fouille. Les sondages répartis sur le pourtour accessible ont permis d'en apprécier les différents types de remplissage.

La taille des fossés de l'enclos est constante. Elle ne varie que très légèrement d'un tronçon à l'autre avec des largeurs oscillant entre 1,20 et $1,40 \mathrm{~m}$. Les profondeurs conservées sont comprises entre 0,50 et $0,66 \mathrm{~m}$. Cette profondeur variant peu, il est possible d'apprécier le sens des écoulements sur la partie de l'enclos qui a pu être topographiée. Les points les plus hauts se situent au niveau de son angle sud-ouest. Le ruissellement se faisait d'une part vers l'angle nord puis vers le sud-est jusqu'au fossé constituant le côté détruit par la viabilisation. De la même façon, le fossé qui marque le côté sud-ouest de l'enclos draine les eaux vers le sud-est.

Les phases de remplissage rencontrées sont toujours très lessivées, rendant les différences entre niveaux très peu mar- quées. La base est occupée par des éléments fins issus de l'altération progressive des parois des fossés et d'un tri gravitaire à partir des phases de remplissage supérieures. Les teintes varient de l'orangé au brun orangé et quelques charbons de bois peuvent exister. La principale phase de remplissage qui comble le reste du fossé est le plus souvent brun gris avec des nodules de manganèse, des graviers de quartz et parfois des zones de limons orangés.

D’une manière générale, les couches rencontrées sont peu organiques. Les charbons de bois y sont rares, excepté dans le fossé sud-ouest à la hauteur de la fosse $\mathrm{F} 57$, où une planche ou un rondin a été retrouvé à l'état de charbon de bois. De la même façon, le mobilier archéologique issu des fossés est peu abondant à la hauteur des bâtiments. Toutefois, quelques nuances peuvent être apportées. Le redressement des coupes réalisées lors de la découverte fortuite du site a été l'occasion de recueillir d'assez nombreux nodules et blocs de terre cuite. Il en est de même pour le fossé de partition 

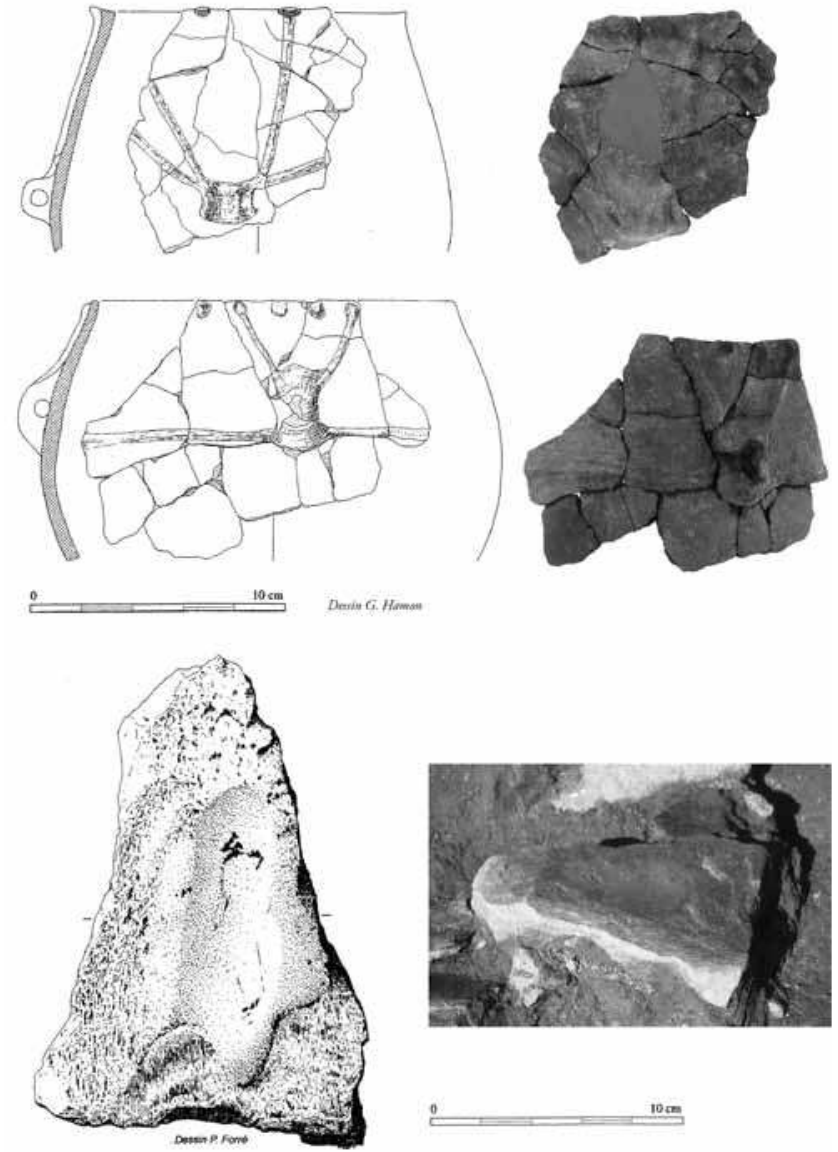

Figure 4 : Une partie du mobilier néolithique découvert dans la fosse F. 40 (2 poteries VSG et un petit polissoir).

Figure 4: Selection of the Neolithic assemblage from pit F 40 (2 VSG pots and a small stone polisher).

interne $\mathrm{F} 44$. Ce dernier a d'ailleurs été reconnu grâce à sa teneur en éléments indurés. Nulle part ailleurs dans les fossés nous n'avons retrouvé ce phénomène.

\section{Les fossés internes à l'enclos}

Deux fossés ont été découverts dans l'enclos de La Tène (F 44 et F 32, fig. 5). Leurs orientations cohérentes avec celles de l'enclos, leur localisation dans l'enceinte, et leurs interruptions permettent probablement de les associer à un système de partition interne.

Le fossé $\mathrm{F} 44$ a une orientation sud-ouest/nord-est. Il a été suivi sur $24,50 \mathrm{~m}$. Au sud-ouest, il s'interrompt à un peu plus de $3 \mathrm{~m}$ du fossé sud-ouest de l'enclos et au nordest nous perdons sa trace au-delà de la limite décapée. En coupe, sa lecture est difficile. Son niveau d'apparition a pu être localisé juste au-dessous de l'humus grâce aux éléments de terre cuite qu'il contenait. Ce fossé n'a pas livré d'autre mobilier. En coupe, il mesure 0,3 à $0,4 \mathrm{~m}$ de profondeur pour 0,80 à $1 \mathrm{~m}$ de largeur. Son profil montre, comme à son extrémité nord-est, un fond plat et des bords évasés. En dehors des tronçons les plus riches en mobilier, rien du fossé n'était visible, absence de contraste qui pourrait également en expliquer des lacunes de repérage. La découverte, dans son remplissage, de nodules de terre cuite identiques à ceux découverts ailleurs sur le site permet peut-être d'associer cette limite à l'organisation générale de l'enclos. Malgré tout, l'orientation de ce fossé diffère sensiblement de celle de l'enceinte et nous devons admettre qu'il serait hasardeux de vouloir les associer à tout prix.

Un autre fossé (F 32) a été relevé un peu plus au nordouest. Comme pour le précédent, cette limite, difficile à voir au décapage, n’a été relevée que ponctuellement. Suivie par intermittence sur un total de $21 \mathrm{~m}$, elle s'inscrit assez nettement dans les principales orientations de l'enclos. Conservé sur moins de $10 \mathrm{~cm}$ de profondeur, ce fossé atteint une cinquantaine de centimètres de largeur. Son remplissage n'a pas livré de mobilier.

Cette probable limite parcellaire n'a pas de contact avec le fossé sud-ouest de l'enclos, sans que l'on sache s'il s'agit d'une interruption réelle ou simplement due à son niveau d'arasement. À son autre extrémité en revanche, quatre trous de poteau, qui forment un ensemble cohérent, confirment la présence d'une interruption. Enfin, si l'on restitue la partie manquante de l'enclos, cette limite le divise en deux parties presque égales.

\section{Un réseau parcellaire associé?}

Le fossé F 58 se greffe au niveau de l'angle ouest de l'enclos. Cette limite, orientée nord-ouest/sud-est, a été suivie sur $14 \mathrm{~m}$ de longueur. Elle mesure $0,90 \mathrm{~m}$ de largeur et presque $0,30 \mathrm{~m}$ de profondeur. Son profil à fond plat montre des bords évasés et aucun mobilier n'a été recueilli dans l'unique sondage ouvert. Sa connexion au niveau d'un angle de l'enclos permet de l'associer à un même complexe. La coupe réalisée au contact de ces deux limites excavées n'a pas permis d'observer de différences entre des phases de remplissages totalement lessivés.

Le fossé F 224. Repéré dès l'évaluation et décapé plus largement à l'occasion de la fouille, ce fossé rectiligne a été reconnu sur $35 \mathrm{~m}$ de longueur parallèlement au côté sud-est de l'enclos. Il mesure 0,75 à $0,95 \mathrm{~m}$ de largeur pour 0,17 à $0,34: \mathrm{m}$ de profondeur. Avec une orientation qui s'écarte nettement des limites parcellaires du cadastre napoléonien, cette limite semble bien appartenir au même ensemble que l'enclos, ce qui semble confirmé par quelques tessons de La Tène finale issus de son remplissage. Ce fossé pourrait ainsi doubler le côté sud-est de l'enclos.

Plus au sud, d'autres fossés pourraient également faire partie de cet ensemble. 


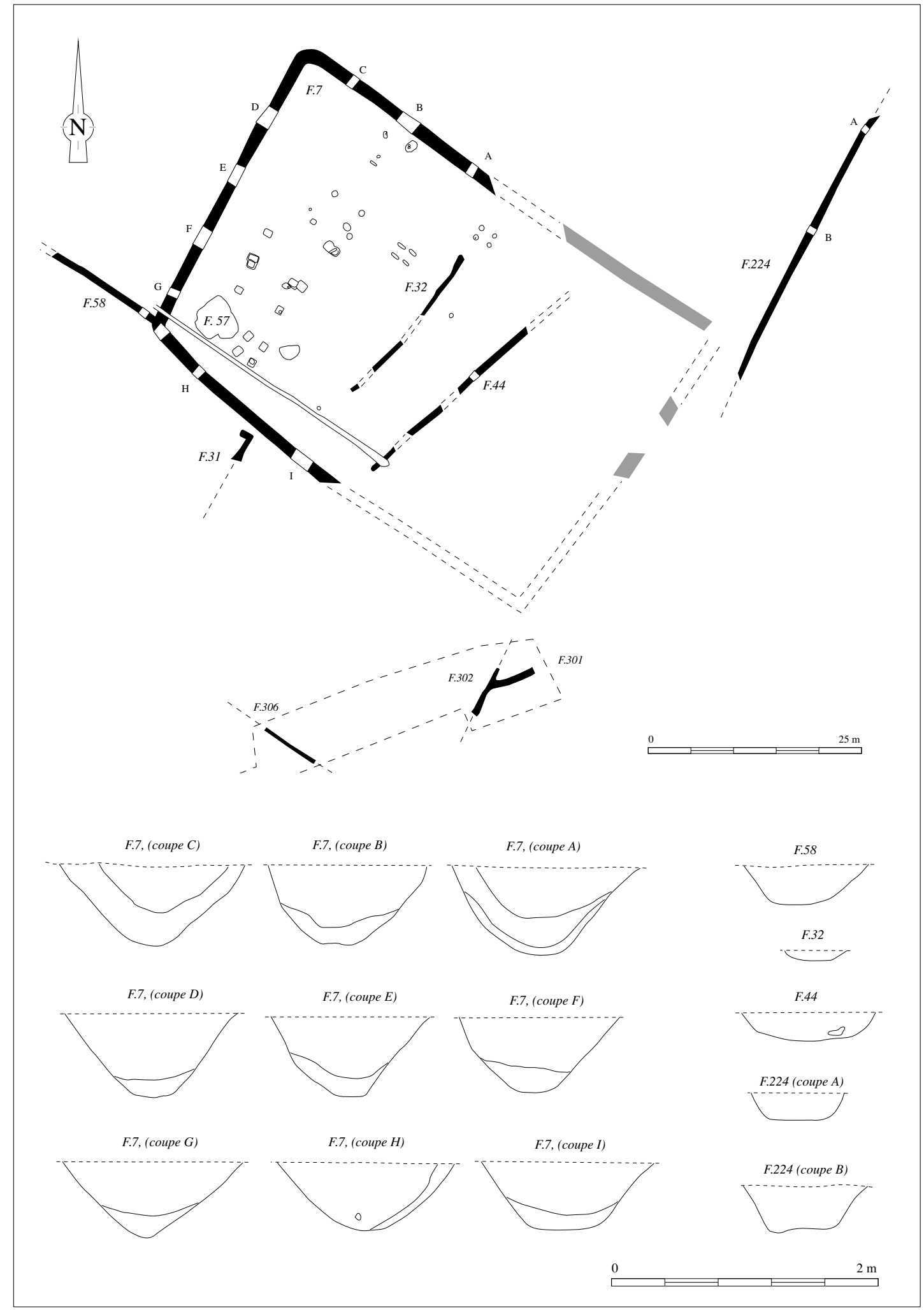

Figure 5 : Plan du site de La Tène finale avec les profils des fosses relevés depuis le niveau décapé (environ 0,50 $\mathrm{m}$ sous la surface actuelle).

Figure 5: Plan of the late La Tene settlement, and profiles of ditches (drawn from clearance level, nearly $50 \mathrm{~cm}$ under modern surface). 
- Le fossé F 306 mesure $0,45 \mathrm{~m}$ de largeur pour $0,10 \mathrm{~m}$ de profondeur. Il a été suivi sur $8 \mathrm{~m}$ de longueur. L'orientation de cette limite s'apparente à celle du fossé sud-ouest de l'enclos principal.

- Le fossé $\mathrm{F} 302$, dans ce même sondage, mesure de 0,60 à $0,90 \mathrm{~m}$ de largeur pour 0,22 à $0,32 \mathrm{~m}$ de profondeur. Suivi sur $8 \mathrm{~m}$ de longueur, il s'interrompt au nord-est, recoupé par un fossé du parcellaire actuel. Il n'a pas livré de mobilier. Perpendiculaire au fossé F 306, il semble ainsi aligné sur l'angle sud de l'enclos.

- Un autre fossé, F 301, vient se greffer sur le précédent. Suivi sur $6 \mathrm{~m}$ de longueur, large de $0,90 \mathrm{~m}$ et profond d'une quarantaine de centimètres, il n'a pas livré de mobilier. Son orientation s'écarte assez nettement du reste des fossés attribuables à La Tène même si le contact avec F 302 montre qu'ils ont au moins fonctionné un temps ensemble.

- Le fossé $\mathrm{F} 31$, qui se termine en angle droit à $0,90 \mathrm{~m} \mathrm{du}$ fossé sud-ouest de l'enclos, pourrait faire également partie du même complexe du fait de son orientation. Il mesure $0,80 \mathrm{~m}$ de largeur et il a été suivi sur 3,50 $\mathrm{m}$ de longueur avant qu'il ne sorte du décapage.

Les autres limites fossoyées mises au jour ont été retrouvées sur les planches du cadastre napoléonien; elles ont toutes des orientations différentes de celles du complexe protohistorique (fig. 6).

\section{Les autres Structures de La Têne}

\section{Les trous de poteaux}

\section{Généralités, répartition et conservation}

Les trous de poteaux et plus généralement le reste des structures en creux restent à distance des fossés de l'enclos (fig. 5). L'absence de creusement dans l'axe du site et dans sa moitié orientale, pour ce que nous en savons du moins, est à souligner.

La conservation des structures est moyenne comme le montre la coupe du fossé F 44. Le niveau d'apparition des creusements, quand ils sont riches en mobilier, se situe en effet à une vingtaine de centimètres au-dessous du niveau de décapage. Pour ceux qui sont pauvres en mobilier, aucune différence n'est visible entre les phases de remblais et les schistes altérés à ce niveau de décapage. Il faut encore descendre jusqu'au substrat non perturbé pour que se dessinent les structures en creux. D'autre part, le décapage a été réalisé à la fin de l'été et dans un premier temps, la sécheresse du substrat a empêché de discerner toutes les anomalies archéologiques. C'est ainsi que la première semaine d'intervention s'est attachée à ne tester que les structures évidentes comme les fossés et les fosses les plus vastes. L'arrivée de la pluie nous a ensuite permis de retrouver d'autres creusements.
Un secteur montrant de nombreuses anomalies autour de la fosse F 57 a fait l'objet d'un premier relevé. Ces taches informes, qui ne semblaient n'être liées qu'à des phénomènes de bioturbation, ont été testées. Ces sondages ont montré que certaines d'entre-elles masquaient en fait des trous de poteaux.

\section{Deux bâtiments associés à une même phase de construction}

Un bâtiment rectangulaire.

Un ensemble de structures de plan comparable dessine un rectangle de 8,20 m sur 6,10 m (fig. 6). Localisé dans la partie ouest de l'enclos, ce qui apparaît nettement comme un bâtiment est parallèle au fossé occidental. Cette construction est délimitée par les vestiges de trois trous de poteaux, F 2, 52 et 53, de deux doubles trous, F 50/68 et 55/54, et peutêtre d'un sixième, F 67. Tous ont des plans rectangulaires; leurs longueurs varient de 1,08 à $1,30 \mathrm{~m}$ pour des largeurs qui fluctuent de 0,70 à $0,90 \mathrm{~m}$ (fig. 7). Les profondeurs sont plutôt régulières puisqu'elles sont comprises entre 0,34 et $0,40 \mathrm{~m}$. Ces creusements ont perforé la couche de limon jusqu'à atteindre, sans l'attaquer, la surface des schistes.

Les trous simples (F 2, 52, 53) sont de gabarit extrêmement régulier. Ils occupent trois des angles de l'espace. Les trous composites sont placés au milieu des grands côtés du rectangle. Les relations observées à la fouille et les observations faites sur les contacts entre avant-trous indiquent que, dans le cas de F 50/68, le premier est le plus ancien. Cette observation réalisée en coupe semble confirmer le positionnement de F 50, qui est centré par rapport à F 53 et F 52 . Pour l'autre paire, la construction se composant à l'origine de deux alignements de trois poteaux, il semble logique que le poteau $\mathrm{F} 55$ soit postérieur à $\mathrm{F} 54$, mais seule la position du premier hors du plan initial de la construction vient appuyer cette proposition.

Ne subsiste du dernier angle de la construction qu'une simple encoche découverte sur un des versants du fossé moderne F 60. Sa présence est confirmée par l'altitude de l'avant-trou F 67, comparable à celles des autres. L'implantation des restes de ce creusement semble indiquer qu'il en existait un second à proximité, totalement oblitéré par le fossé $\mathrm{F} 60$. En suivant le même raisonnement que précédemment, cet avant-trou manquant limiterait également l'emprise initiale du bâtiment. Trois de ces structures ont livré les traces de poteaux, de diamètres compris entre 0,30 et $0,40 \mathrm{~m}$. Certaines de ces traces n'étaient visibles qu'en coupe.

\section{Un bâtiment carré à proximité.}

Quatre trous de poteaux (F 56, 65, 64 et 61) délimitent un espace rectangulaire de 2,90 sur $3,30 \mathrm{~m}$ (fig. 6). Les avanttrous ont des formes et des dimensions voisines (fig. 8). Ils 
Figure 6 : Les trous de poteau et les fosses de l'enclos avec en grisé les fossés modernes

Figure 6: Postholes and pits inside the enclosure (dimmed structures are modern).

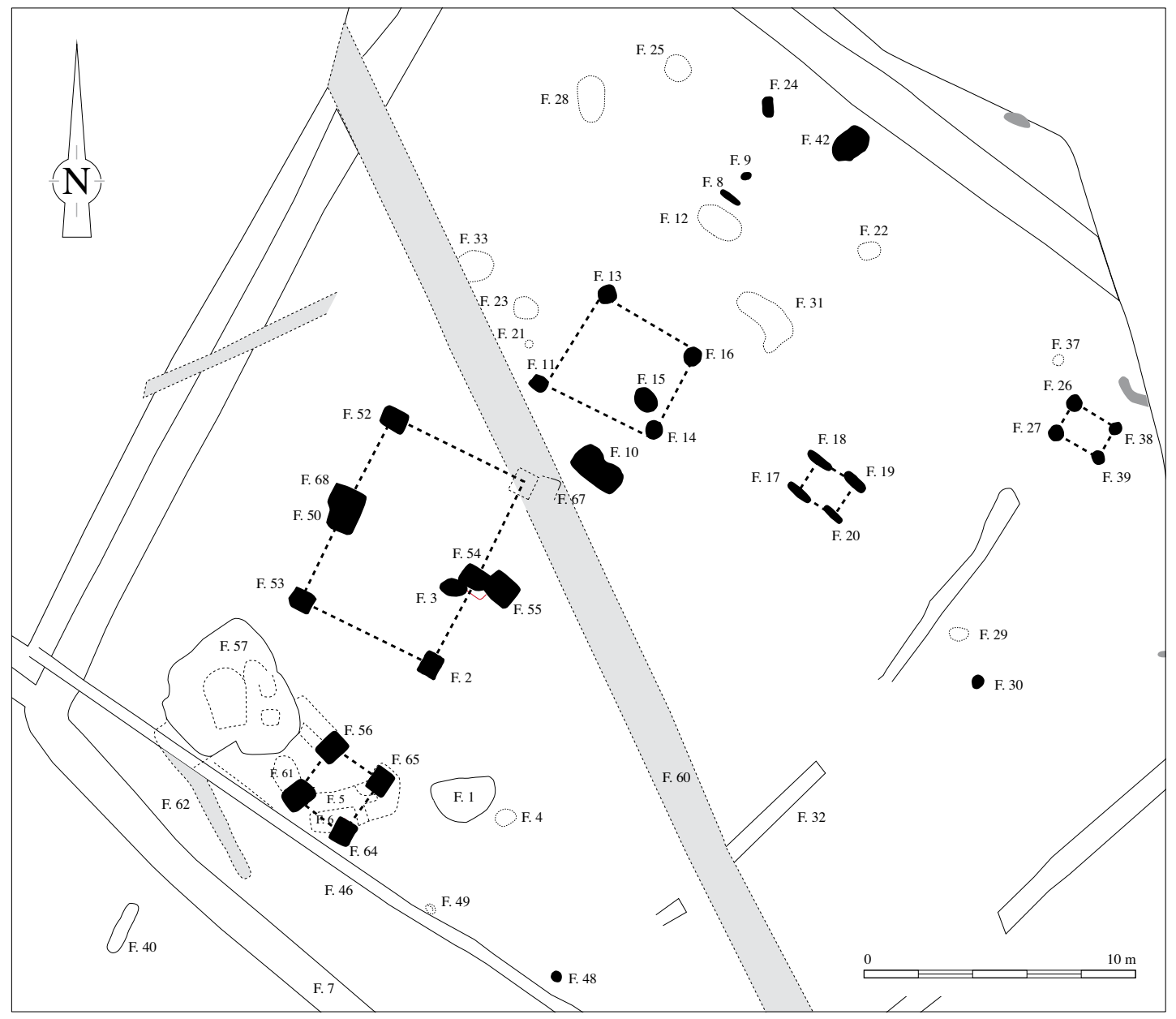

Figure 7 : Plans et profils des trous de poteau du bâtiment rectangulaire relevés au niveau du décapage

Figure 7: Plan and section of the rectangular building postholes (as surveyed from the cleaning surface).

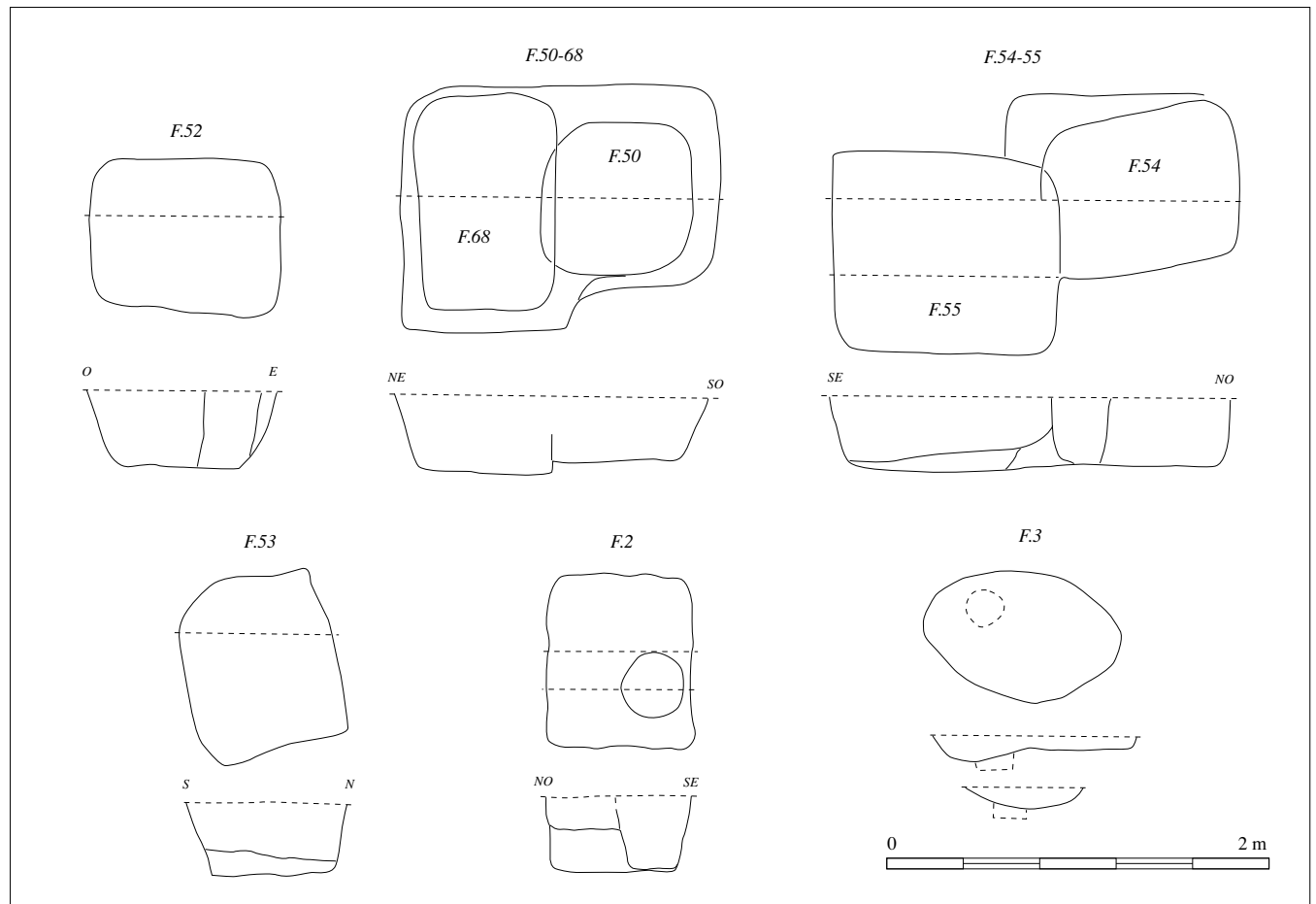




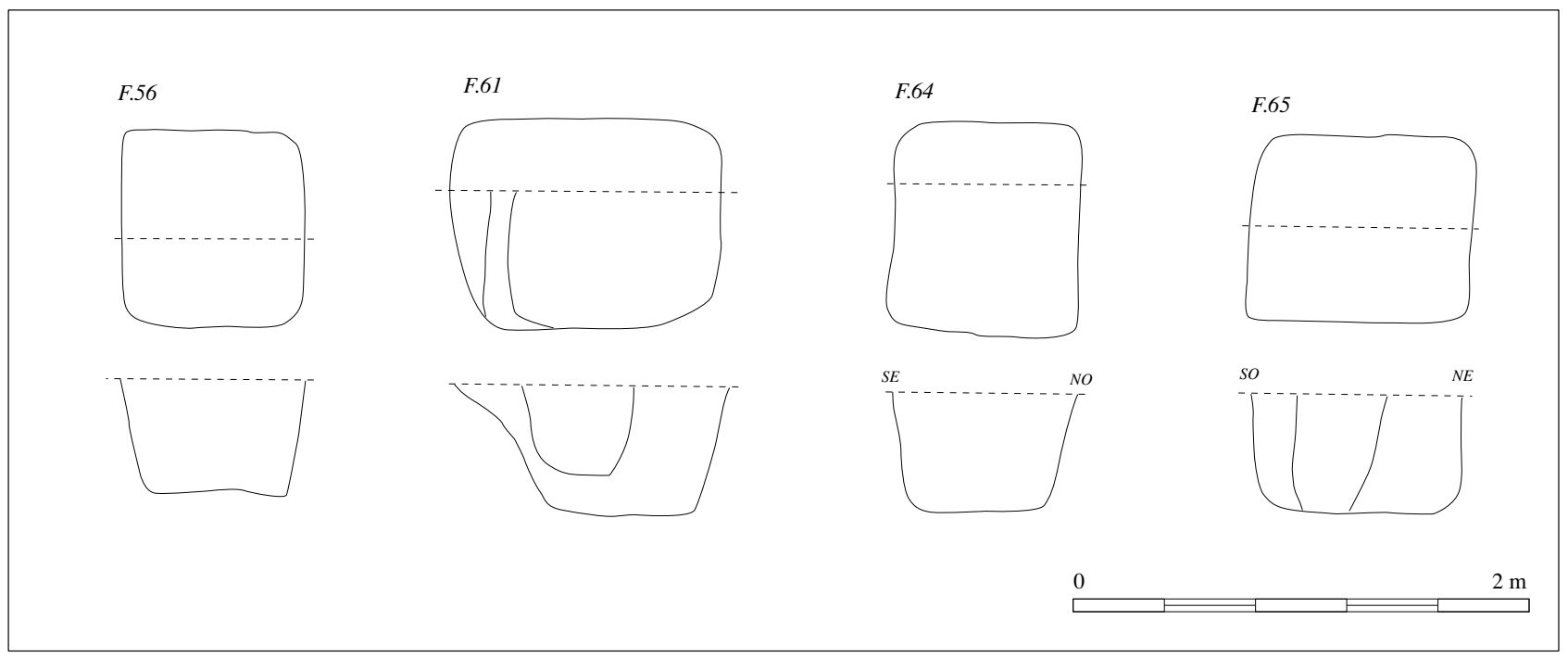

Figure 8 : Plan et profil des trous de poteau du bâtiment carré relevés au niveau du décapage.

Figure 8: Plan and section of the square building postholes (as surveyed from the cleaning surface).

sont également de forme rectangulaire, de 0,80 ou $0,90 \mathrm{~m}$ de largeur pour 0,90 à $1,20 \mathrm{~m}$ de longueur. Les profondeurs atteignent 0,50 à $0,55 \mathrm{~m}$. Pour ces creusements également, les niveaux de limon d'altération ont été perforés jusqu'aux schistes plus durs. Dans deux des quatre structures, des négatifs de poteaux ont été relevés. Ils mesurent entre 0,34 et $0,45 \mathrm{~m}$ de diamètre. Quelques-uns de ces trous ont livré du mobilier de La Tène finale, notamment F 56.

Remarques concernant ces deux bâtiments.

Les parentés entre les deux constructions sont évidentes. La forme des avant-trous, leur taille et dans une moindre mesure leur profondeur, la proximité des deux bâtiments, leur orientation voisine indiquent qu'ils remontent très probablement à une même phase de construction. Ce sont deux bâtiments robustes dont le plan est réalisé à l'aide d'avanttrous de poteaux de gabarit régulier. Avec près de $1,10 \mathrm{~m}$ de profondeur à atteindre pour implanter des poteaux de 0,30 à $0,40 \mathrm{~m}$ de diamètre, il fallait des creusements suffisamment spacieux pour s'y mouvoir à mesure que le travail avançait. Le plan précis des bâtiments n'était ajusté qu'ensuite avec la pose des poteaux, qui ne se trouvaient pas forcément au milieu des excavations.

Les ressemblances techniques entre ces deux bâtiments permettent également de confirmer la fonction du plus grand. Il nous semble qu'avec $51 \mathrm{~m}^{2}$ d'emprise et son implantation dans l'enclos, il ne peut constituer qu'une habitation. En élévation, celle-ci se composait de deux murs gouttereaux à trois poteaux. Les supports qui se répondaient d'un alignement à l'autre devaient être reliés par une sablière haute et par des entraits, ensemble qui permettait sans doute de soutenir un plancher, comme les diamètres des poteaux utilisés l'indiquent (entre trente et quarante centimètres). Aucune information n'a en revanche été recueillie quant à l'emplacement de l'entrée. Le côté sud-est peut néanmoins être privilégié : il est à l'abri des vents dominants et ouvre directement sur le reste de l'enclos.

Pour le second bâtiment, l'hypothèse d'un grenier est privilégiée (fig. 9). Le gabarit des supports (au-delà de $0,35 \mathrm{~m}$ de diamètre) permet également de restituer une construction imposante. Au-delà de l'hypothèse proposée ici, pourquoi ne pas imaginer un plancher dont l'assise serait légèrement supérieure à l'emprise délimitée par les quatre poteaux? Ce parti aurait l'avantage de fournir une surface utile plus importante. La pose de pierres plates au sommet des supports, élément que l'on ne retrouve jamais en fouille, ne serait pas nécessaire, le surplomb formé par le débordement de la plate-forme étant suffisant pour empêcher les montées de rongeurs...

\section{D'autres petits bâtiments}

Le premier ensemble est constitué des trous de poteaux F 26, 27, 38 et 39 (fig. 6 et 10). Ils ont été relevés pour les deux derniers dans un des sondages de l'évaluation et pour les deux autres à l'extrême est du décapage. En plan, F 26 et 27 ont des diamètres légèrement supérieurs à $0,60 \mathrm{~m}$ pour 0,12 à $0,22 \mathrm{~m}$ de profondeur alors que les deux autres, moins profondément conservés ( $\mathrm{du}$ fait d'un niveau de décapage inférieur de la tranchée d'évaluation), ne mesurent que $0,50 \mathrm{~m}$ environ pour 0,12 à $0,18 \mathrm{~m}$ de profondeur. Ces quatre structures limitent un espace rectangulaire de 2,50 sur $2 \mathrm{~m}$ dont l'orientation est la même que l'orientation générale 


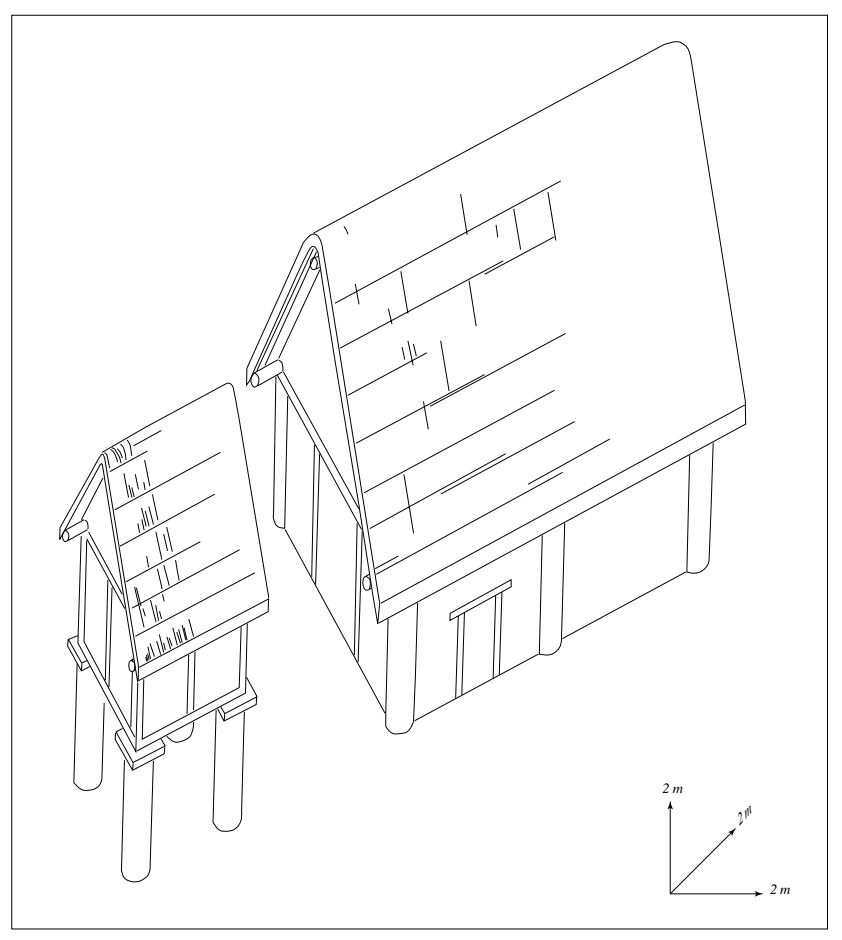

Figure 9 : Évocation en axonométrie de l'ensemble formé par les deux principaux bâtiments du site (dessin E. Mutarelli, INRAP) Figure 9: Axonometric evocation of the grouped two mains buildings.

de l'enclos. La surface modeste de ce petit bâtiment composé de quatre poteaux évoque également un grenier.

Légèrement plus à l'ouest, un second ensemble ressemble également à ce type de construction. Les quatre structures, F $17,18,19$ et 20 , ont des formes singulières. Il ne s'agit pas de trous de poteaux de forme habituelle mais de petites fosses oblongues qui mesurent entre 0,88 et $1,08 \mathrm{~m}$ de longueur pour des largeurs oscillant entre 0,29 et $0,40 \mathrm{~m}$. Les profondeurs sont comprises entre 0,04 et $0,10 \mathrm{~m}$. Cet ensemble délimite un espace de 2,50 m sur $2 \mathrm{~m}$. Les creusements allongés ont exactement la même orientation. Les concordances de plan et d'orientation avec le grenier précédent permettent de l'en rapprocher. Malgré des structures de formes atypiques, il est difficile de ne pas penser qu'il puisse s’agir également d'un grenier.

\section{Les autres trous de poteaux}

Au nord-est du bâtiment principal, une modeste concentration de creusements peut être signalée (fig. 6). Plusieurs trous de poteaux comme F 11, F 13 ou F 16 montrent des profondeurs comprises entre 0,18 et $0,50 \mathrm{~m}$ et des diamètres de 0,65 à $0,70 \mathrm{~m}$. Le trou $\mathrm{F} 16$ présente même l'ultime vestige d'un poteau de presque $0,30 \mathrm{~m}$ de diamètre. Ces trois creusements décrivent un angle droit dont l'orientation générale est comparable à celle déjà remarquée pour les autres bâtiments. Par leur gabarit, ces vestiges ont fait probablement partie d'un autre bâtiment dont il ne subsiste que quelques éléments. Les écartements entre les avant-trous F 11 et F 13 d'une part et F 13 et F 16 d'autre part atteignent $4 \mathrm{~m}$ d'axe en axe, distance proche de celle utilisée dans le bâtiment principal. À cet ensemble, nous hésitons à associer une quatrième structure, $\mathrm{F} 14$. En comparaison des autres ensembles, celui-ci nous semble bien irrégulier.

Une petite tranchée F 8, du type de celles qui constituent l'un des greniers du site, existe également plus au nord-est. Ce creusement a exactement le gabarit et l'orientation des quatre structures F 17, 18, 19 et 20.

Même si ces vestiges de construction sont rares et mal conservés, ils permettent néanmoins de montrer que des bâtiments existaient sans doute sur toute la largeur de cette partie nord occidentale de l'enclos.

\section{Les fosses}

La fosse $\mathrm{F} 1$ a une forme ovalaire (fig. 11). Elle mesure 2,30 m de longueur sur 1,70 $\mathrm{m}$ de largeur pour une profondeur de $0,55 \mathrm{~m}$. Ses bords sont évasés et son remplissage, composé de trois couches, est issu d'activités domestiques, notamment de vidanges de foyer. La couche la plus intéressante, en position terminale de comblement, renfermait, en plus de quelques tessons protohistoriques, des nodules de terre cuite et des fragments de charbon de bois assez nombreux. L'hypothèse d'une fosse de construction, nécessaire la réalisation des parois des bâtiments, est envisageable pour ce creusement voisin des principales constructions.

La fosse F 10, localisée à proximité du pignon nord du bâtiment principal, a une forme sub-rectangulaire. Elle mesure $2 \mathrm{~m}$ de longueur pour 1,10 à $1,30 \mathrm{~m}$ de largeur et 0,10 à $0,20 \mathrm{~m}$ de profondeur. Son remplissage se compose de trois phases. La plus riche d'enseignements est la phase terminale, qui renfermait de nombreux éléments de terre cuite (fragments de parois de structures de combustion), accompagnés de tessons de céramique en assez grand nombre, associés à un mélange de limon et de pierres brûlées. La fonction de dépotoir peut probablement être privilégiée pour une telle fosse, située à proximité immédiate d'une habitation. Il pourrait également s'agir à l'origine d'une fosse de construction.

La fosse F 57 a été relevée juste dans l'angle sud-ouest de l'enclos, entourée par les deux bâtiments principaux. Le creusement mesure $5 \mathrm{~m}$ de longueur sur $4 \mathrm{~m}$ de largeur. En dehors de son côté sud, où plusieurs perturbations sont venues le recouper, ce creusement présente une emprise régulière. La profondeur maximale se trouve au centre de la structure où les schistes ont été atteints à une cinquantaine de centimètres sous le niveau du décapage. Parmi les trois phases de remplissage relevées, la seconde a livré plus de 


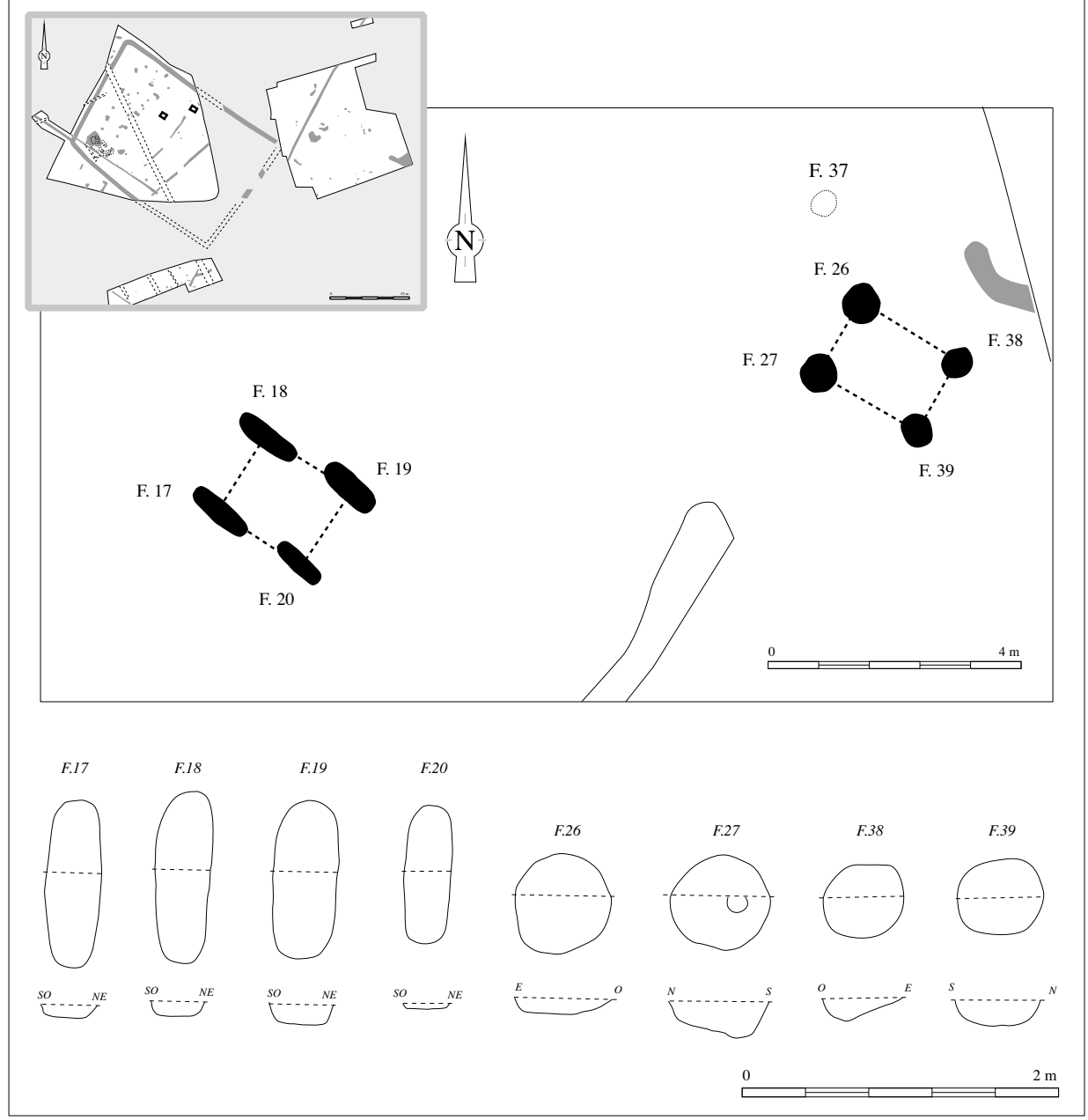

Figure 10 : Les deux petits bâtiments les plus orientaux : plans et profils de leurs structures Figure 10: The two eastern small buildings : plans and sections of their features.
$25 \mathrm{~kg}$ d'éléments d'argile indurée issus pour la plupart de plaques foyères. On peut supposer que ce creusement, voisin des principaux bâtiments, a pu servir dans un premier temps de fosse de construction puis, comme sans doute les deux autres fosses, qu'il a ensuite reçu les rejets domestiques issus des bâtiments tout proches.

\section{LE MOBILIER}

\section{Les données de la céramique (J.-C. Meuret)}

Le nombre total des tessons s'élève à 242 , chiffre dont la modestie surprend pour un site d'habitat et pourrait restreindre la portée des conclusions; mais on verra qu'en raison de la très grande homogénéité du lot, ce n’est pas le cas.

\section{Les pâtes et les cuissons}

75 à $80 \%$ des tessons présentent une cuisson en atmosphère réductrice. Un certain nombre montre cependant des surfaces plus claires, brun-ocre et rarement rouge brique.
Une pâte particulière doit être signalée, de couleur brun-vert sombre avec des mouchetures de surface noires, correspondant sans doute à une argile particulière.

Les cassures reflètent l'irrégularité de la cuisson : elles peuvent être à cœur sombre, brun-brique, noir, quelquefois gris mais sont rarement régulières dans toute l'épaisseur. Un cas seulement montre une structure légèrement feuilletée (lot 23, tesson non dessinable).

Tous les tessons présentent un dégraissant de quartz mais de calibre irrégulier atteignant parfois $10 \mathrm{~mm}$ (lot 10) pour les tranches épaisses. On a plusieurs fois relevé la présence de mica en paillettes très fines et parfois de chamotte (lot 15 : fig. $12 \mathrm{n}^{\circ} 4$ et $5 ;$ lot $\left.10: \mathrm{n}^{\circ} 8\right)$.

Le surfaçage manque de finition : parfois il se résume à des stries multiples de tournage rapide, parfois - et c'est le cas le plus fréquent- il présente un aspect râpeux produit par un surfaçage grossier et rapide. On n'a par exemple observé aucun cas de lustrage. Quant à la fonction des vases, on note dans quelques cas la présence de traces de suie à l'extérieur des panses, signe de leur usage pour la cuisson (lot 10, tessons non dessinables; lot 20 : fig. $12, \mathrm{n}^{\circ} 7$; lot $27: \mathrm{n}^{\circ}$ 9). 


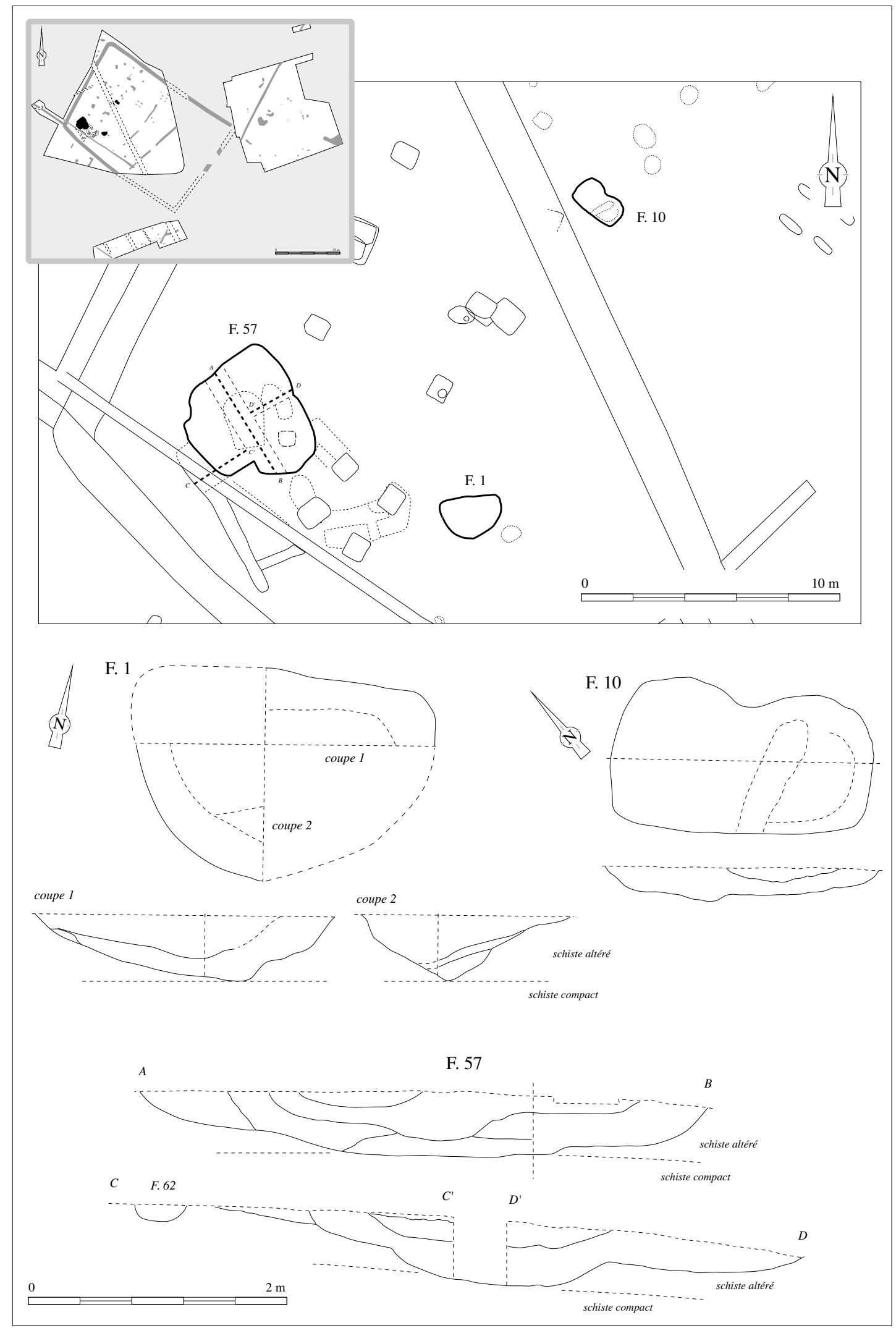

Figure 11 : Les fosses (F. 1, 10 et 57) : leur localisation, plans et profils à partir du niveau du décapage Figure 11: F 1, F 10 and F 57 pits: location, plans and sections (surveyed from the cleaning level). 


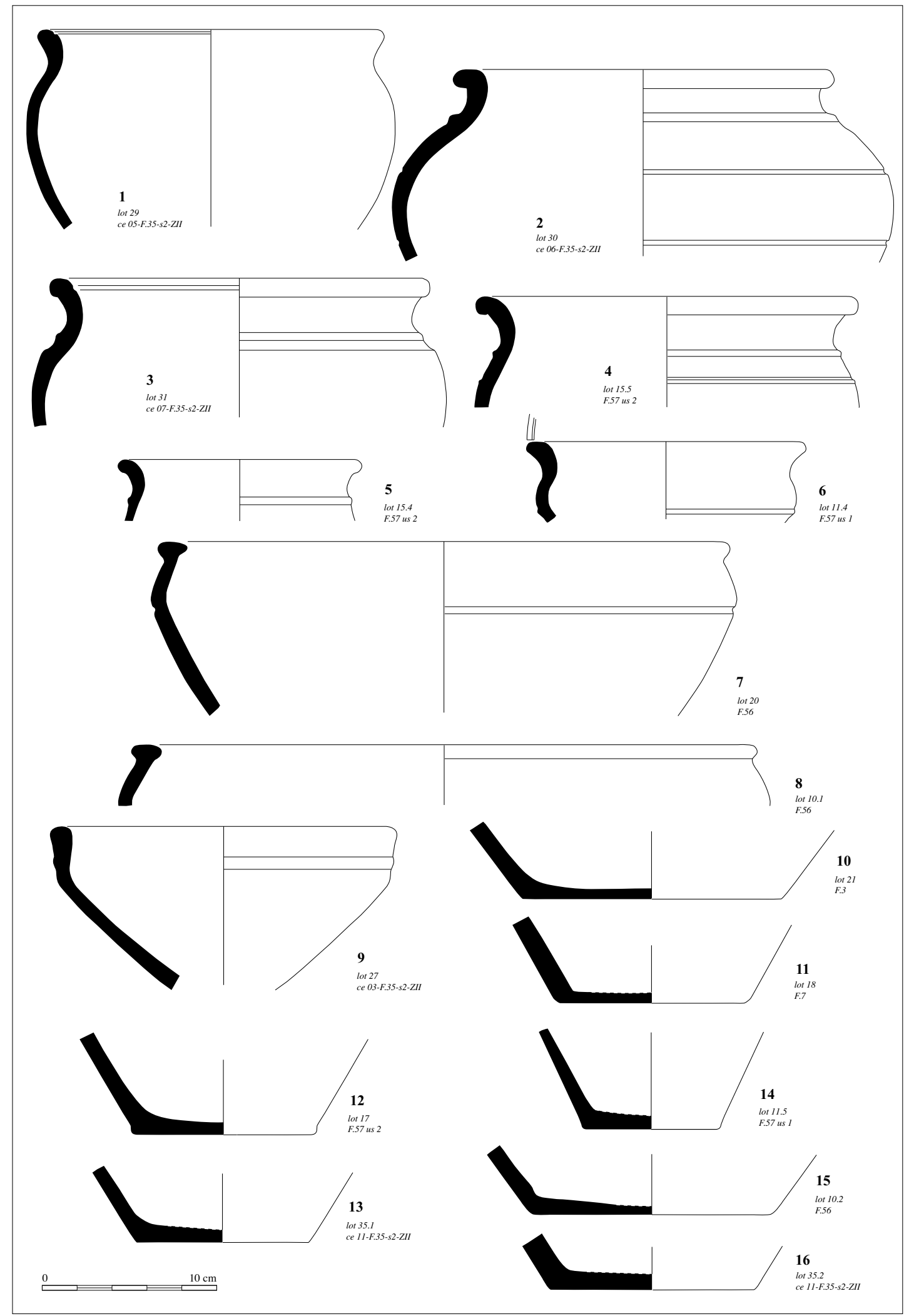

Figure 12 : Le mobilier céramique laténien (dessins J.-C. Meuret).

Figure 12: La Tene ceramic assemblage. 
Tous les tessons appartiennent peu ou prou au même faciès régional à une exception près, celle d'un tesson de couleur rosée provenant du sondage 2, ZII F35 (lot 39 : fosse F 57). M. Monteil, familier des ensembles céramiques du Midi et à qui il fut d'abord présenté, l'identifia comme une céramique à pâte claire fabriquée au sud et donc importée. Pour cette époque, hormis les amphores, les importations de céramique méridonale brillent par leur rareté au nord de la Loire : le fragment d'olpe ampuritaine qui fut identifié à Plouer-surRance (Côtes-d'Armor) dans un contexte du $\mathrm{II}^{\mathrm{e}}$ siècle av. J.-C. demeure une exception (Ménez, 1996, p. 138-139). C'est pourquoi le tesson du Clos-Henry a été soumis à $\mathrm{M}$. Py, autorité indiscutable en la matière, que nous tenons à remercier pour son expertise :

"Le fragment [...] correspond à un vase en céramique tournée à pâte calcaire, couramment désignée sous le vocable de céramique à pâte claire. Les traces de tournage visibles à l'intérieur, l'inclinaison du fragment, ainsi que son diamètre et son épaisseur, permettent d'identifier la base de la panse d'une cruche.

La pâte, de couleur jaune-rosé, contient un fin dégraissant sableux où se remarquent des inclusions de couleurs diverses, certaines blanches et brillantes pouvant correspondre à du mica ou du quartzite, d'autres noires ou brun foncé d'identification plus délicate en l'absence d'une analyse pétrographique. L'épiderme extérieur était lisse à l'origine; l'épiderme intérieur est relativement dégradé.

Le type de vase en question, dans un contexte préaugustéen (Tène finale) et dans une région où ce type de production ne semble pas connu à cette époque à l'échelle régionale, pourrait tout à fait correspondre à une importation méditerranéenne. Les caractères techniques excluent qu'il puisse s'agir d'une production à pâte claire marseillaise. L'aspect sableux de la pâte n'est par ailleurs guère compatible avec les productions locales de la Gaule méditerranéenne, qui d'une manière générale présentent des pâtes plus épurées et plus savonneuses au toucher.

Cette inclusion de sable fin évoque par contre d'assez près les productions de céramiques communes à pâte claire italiennes des II -I rer siècle av. n. è., qui accompagnent dans les épaves les amphores et les mortiers italiques. L'une des hypothèses envisageables, sous toutes réserves évidemment, pourrait être celle d'une cruche d'origine italienne importée en accompagnement des amphores italiques".

\section{Les formes}

Le nombre de vases a été calculé à partir des lèvres et le NMI s'établit à 42, ce qui est important par rapport au faible total de tessons (fig. 13).

Parmi ces lèvres, 37 présentent un profil en $S$ plus ou moins marqué avec forme éversée (fig. 14, n 17, 20, 21, $22,23)$, dont neuf avec une cannelure labiale interne; celle- ci ne dépasse pas $1 \mathrm{~mm}$, sauf dans un cas (lot $31: 3 \mathrm{~mm}$ ); c'est une proportion relativement élevée. Sept lèvres présentent la forme caractéristique du bourrelet externe (fig. 12, $\mathrm{n}^{\circ} 2$ et 4 ; fig. $\left.14, \mathrm{n}^{\circ} 25\right)$; enfin, on relève une autre forme caractéristique qui est celle des lèvres de section triangulaire et sans col, en " $\mathrm{Y}$ ", à bourrelet externe et interne et forme aplatie au sommet, mais avec des variantes (lots 10, 15, 20 : fig. $13, \mathrm{n}^{\circ} 7$ et 8 ). Notons enfin un bord particulier, celui d'une céramique fine à profil vertical, sans col, avec une petite lèvre en léger bourrelet (lot 16 : fig. 14, $\mathrm{n}^{\circ} 18$ ).

Parmi les onze fonds, aucun ne présente l'anneau caractéristique des jattes, bien que certaines panses appartiennent à ce type de vase. Tous sont plats et se rapportent donc plutôt à des vases simples de conservation ou de stockage. Les angles de panses mesurés sur ces pieds par rapport à l'horizontale vont de $130^{\circ}$ à $100^{\circ}$ (fig. $12, n^{\circ} 10$ à 16); ceux-ci appartiennent donc à des formes hautes, de type pot. Le diamètre moyen de ces fonds s'établit à $11,4 \mathrm{~cm}$.

Des traces de tournage rapide s'observent sur un vase sur cinq; ce sont des stries multiples, des cannelures et des cordons en haut de panse (fig. 12, nº 2 à 6). Mais la régularité de toutes les formes prouve qu'elles relèvent bien de cette même technique; de plus, aucun tesson ne présente d'irrégularité attribuable à la pratique du modelage.

Hormis les cannelures et cordons qui n'en sont pas vraiment, les décors sont très rares : ni incision, ni impression, ni molette, ni estampage; on note seulement quelques rares stries lissées (lot 26), soulignant le tournage ou perpendiculaires à celui-ci (lot 15-1 : fig. 14, n 19).

Les types de formes générales ont été évoqués plus haut. Ce sont seulement 27 vases au total qui ont pu être déterminés, sachant qu'une seule forme est archéologiquement complète. Les formes basses sont minoritaires, de type jatte ouverte, avec un angle de panse très fuyant, de l'ordre de 30 à $40^{\circ}$. Il en est de même pour les formes moyennes avec un angle de 50 à $60^{\circ}$. Avec plus de la moitié du total, les formes hautes l'emportent donc nettement. La typologie des lèvres amène à conclure à une majorité de vases ouverts ou semiouverts et à la rareté des formes fermées de type globulaire (lots 14, 17 et 24 : fig. 14, nº 24 à 26).

Le diamètre à l'ouverture va de 16 à $24 \mathrm{~cm}$, pour une moyenne de $23,7 \mathrm{~cm}$. Aux extrêmes, il faut signaler un petit vase à paroi fine (lot 16), de $15 \mathrm{~cm}$, et quatre gros exemplaires de 54,50 et $46 \mathrm{~cm}$ de diamètre, qui sont des vases de stockage.

\section{Comparaisons, affinités culturelles, conclusions et proposition chronologique}

L'ensemble céramique recueilli présente une très grande homogénéité, tant dans les cuissons à majorité réductrice que dans les formes, probablement toutes tournées, ou dans 


\begin{tabular}{|c|c|c|c|c|c|c|c|}
\hline NMI/lèvres & Lèvre éversée & $\begin{array}{c}\text { Cannelure } \\
\text { labiale int. }\end{array}$ & Lèvre en « Y » & $\begin{array}{c}\text { Lèvre à } \\
\text { bourrelet }\end{array}$ & Fond plat & $\begin{array}{c}\text { Traces de } \\
\text { tournage }\end{array}$ & $\begin{array}{c}\text { Cordon ou } \\
\text { cann./panse }\end{array}$ \\
\hline 42 & 37 & 9 & 3 & 7 & 11 & 25 & 18 \\
\hline$\% / l$ lèvres & $88 \%$ & $19,5 \%$ & $7,50 \%$ & $16,5 \%$ & & &
\end{tabular}

\begin{tabular}{|c|c|c|c|}
\hline Forme basse & Forme moyenne & Forme haute & Gros vase \\
\hline 7 & 6 & 14 & 4 \\
\hline $26 \%$ & $22 \%$ & $52 \%$ & \\
\hline
\end{tabular}

Total : 242 tessons

Figure 13 : Tableau statistique général pour la céramique laténienne. Figure 13: General statistical chart for La Tene ceramics.

les décors. Pour autant, il convient de nuancer et de comparer avant d'aboutir à des conclusions géographiques et chronologiques.

Considérons d'abord les dimensions des vases. Au ClosHenry, avec 23,7 cm de diamètre à l'ouverture, on se situe au-dessus des moyennes connues pour l'Armorique de la fin de l'âge du Fer, où la majorité va de 11 à $20 \mathrm{~cm}$ (Daire, 1991, p. 37). De plus, on doit tenir comme un fait particulier la présence ici de très grands vases à lèvre très éversée, d'un diamètre compris entre 46 et $54 \mathrm{~cm}$ (fig. 14, n 24 à 26). Or, dans l'Ouest, ni à L'Homme-Mort, ni aux Ebihens, ni au Boisanne, on ne trouve une aussi forte représentation pour de telles dimensions : au Boisanne, deux vases seulement dépassent $40 \mathrm{~cm}$, sur 726 diamètres mesurés (Ménez, 1996, p. 126-127)! De manière générale, les sites bretons ne fournissent guère de ces grands vases (Hinguant et Le Goff, 1998, p. 105). Il faut regarder tout à l'est de l'Ille-et-Vilaine, pour voir apparaitre le phénomène, par exemple sur le site de La Montagne en Visseiche (Meuret, 2006, p. 27). Pour trouver une présence comparable, il faut arriver dans le Maine avec des exemplaires pour Ecorcé à Entrammes et l'oppidum de Moulay (Naveau, 1977, p. IX et x), ou encore La Glanerie à Athée (Meuret, 1997, p. 67). Le fait est plus courant en France septentrionale où une étude récente a montré une présence de grands vases de l'ordre de $10 \%$ (Gransar, 2000, p. 288). Sans doute doit-on mettre ici en relation la fréquence de ces gros vases à provisions avec les autres capacités de stockage qui nous sont révélées sur le site du Clos-Henry par la présence de trois greniers probables au voisinage immédiat du bâtiment principal; on sait en effet qu'à toutes les phases des âges du Fer, le stockage en céramique est complémentaire du stockage en grenier ou silo (Gransar, 2000). À moins qu'il ne faille y voir, pour la haute Armorique, une pratique différente de celle de l'Armorique péninsulaire.

D'autres arguments permettent de distinguer dans ce lot un faciès différent de celui de l'Armorique péninsulaire. Ainsi, aucun tesson graphité n'a été observé au Clos-Henry alors que cette pratique atteint des taux élevés à l'ouest et décroît régulièrement vers l'est. Cette observation est à rapprocher de la présence importante de fragments de plaques foyères au Clos-Henry. Ce type de reste est omniprésent sur les sites de Haute-Armorique comme d'ailleurs du reste de la Gaule, mais absent des sites ouest-armoricains de La Tène. Ces éléments présentent une épaisseur de 4 à $4,5 \mathrm{~cm}$, avec parfois un bord lissé et, dans tous les cas, une cuisson peu poussée. Aucun ne comporte de perforation, contrairement à certains sites comme Retiers (Hinguant et al., 1997, p. 70), Rannée ou Athée (Meuret, inédit), pas plus que de forme particulière comme au Terrain des sports de Jublains (Boissel et Naveau, 1980, pl. 17). Comparé à Rennes-Beaurade, où l'on en a recueilli "près de $20 \mathrm{~kg}$ " (Leroux et al., 1999, p. 186), le site du Clos-Henry, avec un total de $25 \mathrm{~kg}$, se place en bon rang, mais loin derrière Visseiche-La Montagne qui en a déjà produit $171 \mathrm{~kg}$ (Meuret, 2006). Quoi qu'il en soit de leur typologie, on tient avec ces plaques de cuisson mobiles un élément distinctif très marquant entre les cultures domestiques de l'ouest et de l'est de l'Armorique.

Sans doute faut-il aussi rappeler l'absence totale de la jatte à bord rentrant, forme au contraire omniprésente à partir de la Loire et vers le sud de la Gaule (Cornu, 1997, p. 194196). J.-P. Bouvet rappelle que l'abs ence de cette forme céramique doit être mise en relation avec la présence, dans le Maine et le sud de l'Orne, d'une autre forme qui pourrait être son substitut régional (Bouvet, 1997, p. 228-229); nous voulons parler de l'écuelle à bourrelet externe et de ses variantes, bien représentée au Clos-Henry. On en a trouvé sur la plupart des sites mayennais tels Moulay-bourg, le Grand-Mesnil à Moulay, Ecorcé à Entrammes, le terrain de sport à Jublains, le Port-Salut à Entrammes, La Chevalerie à Montflours, La Mazure à Azé (site tout proche du ClosHenry), ainsi que sur les sites inédits des Bozées à Laval, de Gesnes-le-Gandelin et de Teillé dans la Sarthe (Bouvet, 1997, p. 229). À ce type de vase caractéristique de la région, nous ajouterons une autre forme présente au Clos-Henry, 


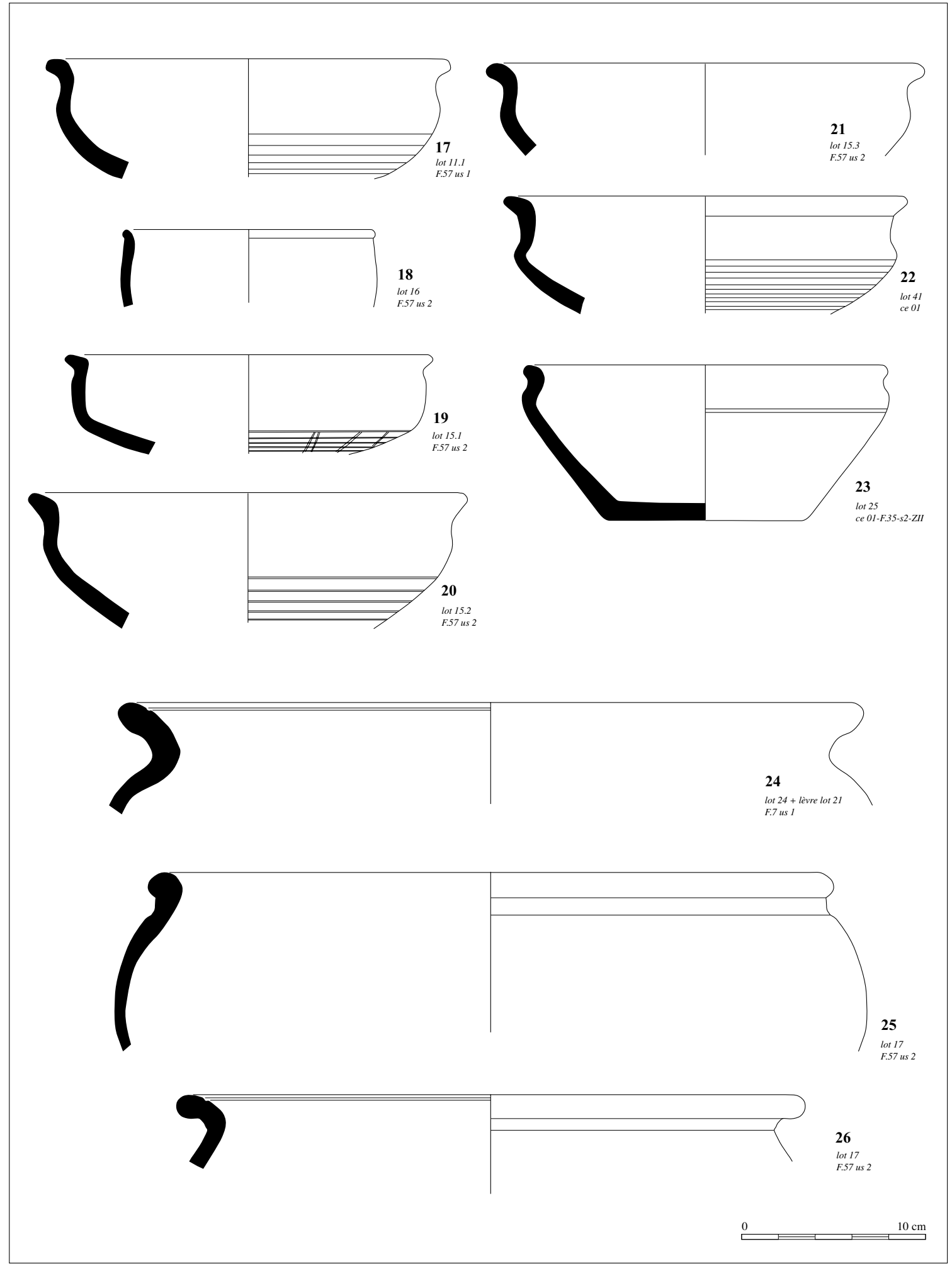

Figure 14 : Le mobilier céramique laténien (suite) (dessins J.-C. Meuret). Figure 14: La Tene ceramic assemblage (continued). 
la lèvre en " $Y$ ", ou encore à section triangulaire sans col (lots $10,15,20$ : fig. $12, n^{\circ} 7$ et 8 ). Elle correspond à des vases tronconiques de forme moyenne. On en connaît en Haute-Bretagne comme aux Jeusseries en Retiers, au Clouet en Carquefou (Le Goff, 2003, p. 111), à La Montagne en Visseiche (Meuret, 2006, p. 26), à La Ligne Anne en Rannée (Meuret, 1992, fig. 5) et on les retrouve avec des variations sur les sites mayennais : l'oppidum de Moulay (Naveau, 1976, pl. XIII), Ecorcé à Entrammes (Naveau, 1977, pl. X), Azé (Lambert et Rioufreyt, 1976, p. 16 et 18). Concernant les signes d'éventuelles influences du sud au Clos-Henry, on observe la présence d'un vase de type jatte carénée (lot 15-1 : fig. 14, n 19), que J.-P. Bouvet a relevé à Angers, aux Pichelots, à Guérande et à Chênehutte-lesTuffeaux (informations orales); mais ce n'est ici qu'un seul exemplaire, tout comme le vase fin à profil vertical (lot 16 : fig. $\left.14, \mathrm{n}^{\circ} 18\right)$ dont on retrouve les équivalents au château d'Angers (Bouvet et al., 2003, p. 182), au Grand-Aunais à Yvré-l'Évêque (Vacher et Bernard, 2003, p. 208). Avec ces vases tronconiques à lèvre en $\mathrm{Y}$ et les lèvres en bourrelet, ajoutés à une plus grande représentation des vases de stockage, à une taille supérieure des vases en général, à l'absence de bords rentrants, à l'absence de graphitage et à la présence de plaques foyères, probablement tient-on là un ensemble de traits qui permettent de commencer à cerner un faciès céramique particulier; celui-ci engloberait l'Ille-et-Vilaine (surtout à l'est), la Mayenne, la Sarthe, le nord du Maineet-Loire et de la Loire-Atlantique. Il se distingue nettement de ce qui se voit à l'ouest de la Bretagne et, plus au sud, le long et au sud de la Loire. Pour autant rien ne permet de transposer ces observations céramiques et très matérielles au domaine culturel général, même si la région définie correspond étrangement à l'extension des mentions épigraphiques gallo-romaines - mais sur fond religieux antérieur - de Mars Mullo.

Pour autant, l'ensemble céramique du Clos-Henry appartient bien encore à l'ensemble typologique armoricain dans la mesure où le taux de cannelures labiales internes y demeure élevé (fig. 12, n 1, 3, 6). C'est là une caractéristique de la céramique armoricaine - au sens géographique et non protohistorique du terme - où les taux vont de $20 \%$ jusqu'à $60 \%$ sur les sites du Finistère, du Morbihan ou des Côtes-d'Armor. On en trouve cependant dans la Manche, le Calvados et même la Seine-Maritime (Daire, 1992, p. 102-104). Cette fréquence sur le site du Clos-Henry n'a donc rien d'anecdotique et dénote une indiscutable appartenance culturelle armoricaine ou tout au moins des courants d'échanges avec cette région. Elle ne fait que renforcer une constatation déjà faite en Mayenne à Azé, Entrammes, Evron, Jublains, Montflours, Moulay (ibid., p. 106-107), ou
Athée (Meuret, 2000, p. 101), dans des proportions importantes mais qui n'ont pas été chiffrées.

Le vaisselier du Clos-Henry relève pour sa quasi-totalité d'une production réalisée au tour rapide. Si l'on se réfère au phasage de la céramique établi par Y. Menez pour le site du Boisanne à Plouer-sur-Rance - le plus proche de ChâteauGontier, l'ensemble du Clos-Henry s'apparente plutôt à sa phase IV (Menez, 1996, 120-122), marquée par " une mâ̂trise complète du tour rapide " et datée de l'ensemble du $\mathrm{I}^{\mathrm{er}}$ siècle av. J.-C. Cependant, la prédominance des formes hautes à Château-Gontier amène à corriger et à vieillir un peu cette datation, par comparaison avec l'Homme-Mort à Saint-Pierre-de-Plesguen (Ille-et-Vilaine) et les Ebihens à Saint-Jacut-de-la-Mer (excavation B), dans les Côtes-d'Armor (Daire, 1991, 40); cela nous amène à placer le lot du Clos-Henry - où jattes et bols représentent moins de la moitié des formes identifiées - autour du début du $\mathrm{I}^{\mathrm{er}}$ siècle av. J.-C.

La cannelure interne de la lèvre fournit un autre indice de datation. Sur le site du Boisanne, aux dernières phases IIIa (250 à 150 av. J.-C.) et IV (100 av. J.-C. à 0), elle se réduit à $1 \mathrm{~mm}$ et même disparaît. Avec les réserves qu'implique une série beaucoup plus courte, il semble qu'au Clos-Henry, où dominent les cannelures de $1 \mathrm{~mm}$ (huit des neuf exemplaires observés), l'occupation ne puisse se placer qu'aux $\mathrm{II}^{\mathrm{e}} \mathrm{ou}$ $\mathrm{I}^{\text {er }}$ siècle av. J.-C. (Menez, 1996, 128).

Aucun reste d'amphore n'a été découvert au Clos-Henry, ce qui nous prive d'une autre source d'information chronologique externe. Cependant on a vu qu'un tesson provient du Sud, sans doute d'Italie, (lot 39) et se place au cours des $\mathrm{II}^{\mathrm{e}}$ ou I $\mathrm{I}^{\mathrm{er}}$ siècle av. J.-C. Cette dernière information chronologique d'origine exogène peut être confrontée sans contradiction à toutes celles fournies par l'examen de la céramique indigène et les comparaisons avec des sites voisins : cannelures labiales nombreuses (plutôt II $^{\mathrm{e}}$ siècle-début I ${ }^{\mathrm{er}}$ av. J.-C.), importance des formes tournées ( $\mathrm{I}^{\mathrm{er}}$ siècle av. J.-C.), finition générale des vases (type Tène finale). Cela nous amène à proposer pour le Clos-Henry, une datation large entre 150 et 50 av. J.-C., peut-être plus resserrée si on se réfère à la clarté et à l'unicité des structures en creux.

\section{Le reste du mobilier}

Dix fragments de pierre brûlée, probablement du grès, issus d'une meule (d'un type non identifiable) ont été découverts dans le fossé $\mathrm{F} 44$ (lot 22). Plus anecdotique sans doute, une pointe de flèche a été recueillie au cours de l'évaluation, au sommet du remplissage du fossé nord-ouest de l'enclos (étude P. Forré, INRAP). Il s'agit d'un exemplaire perçant, à base concave et aileron équarri, en silex blond (secondaire), issu des terrasses de la Loire ou du Loir; cet objet 
peut être attribué au Bronze ancien. Il est intéressant de signaler qu'une autre de ces flèches a été recueillie au cours des sondages réalisés sur le site voisin de la Mazure à Azé. Il s'agissait là d'une pointe de flèche denticulée à ailerons et pédoncules en silex blanc (Lambert et Rioufreyt, 1976, p. 17). Il est difficile de dire s'il s'agit d'un hasard ou d'une récupération opportuniste d'objets anciens encore efficaces et qui auraient été réutilisés par les habitants de ces deux sites de La Tène finale.

\section{SyNTHÈSE DES DONNÉES ET CONCLUSIONS}

\section{Un plan de ferme de La Tène D1 très lisible}

Le site du Clos-Henry est constitué d'un enclos orienté nord-ouest/sud-est qui mesure $40 \mathrm{~m}$ sur 60 environ. Les fossés sont de taille modérée avec $1,30 \mathrm{~m}$ de largeur pour $0,80 \mathrm{~m}$ de profondeur observée environ. En supposant que le sol gaulois était celui de la parcelle avant décapage, ces fossés ne devaient pas dépasser $1,40 \mathrm{~m}$ de profondeur et $2 \mathrm{~m}$ de largeur. En dehors de deux structures (F 24 et F 42) ouvertes à proximité immédiate du fossé nord-est, une bande de 2 ou $3 \mathrm{~m}$ de large le long des fossés de l'enclos est dépourvue de creusements. Cette zone pourrait correspondre à l'emprise d'un talus.

Même si le site n'a pu être totalement appréhendé en raison des destructions liées aux travaux de viabilisation, l'organisation générale de l'enclos semble assez nettement se dessiner. Pour ce que nous en savons, les bâtiments y sont implantés dans la moitié nord-ouest. Le bâtiment le plus vaste, où l'on reconnaît une habitation, reprend exactement l'orientation du fossé nord-ouest. Comme généralement, l'habitation est établie à l'abri d'un talus. Implantée à proximité immédiate du grand axe de l'enclos; elle en constitue, par sa position centrale, l'élément privilégié. Ce bâtiment est également associé à un grenier principal; les similitudes remarquées entre leurs structures permettent sans doute d'en attribuer la construction à une même phase voire à une même équipe. Deux autres greniers d'importance plus modeste complètent cet ensemble.

\section{Le plan de la ferme du Clos-Henry dans son contexte régional}

Le type d'enclos ainsi mis au jour évoque de nombreuses autres enceintes reconnues en photographie aérienne par G. Leroux (fig. 15). Notre collègue insiste sur le nombre important de ces enclos simples à partition interne, notamment dans le sud-ouest du département de la Mayenne. Plusieurs d'entre eux, souvent plus vastes que le Clos-Henry, y ont été repérés, comme celui des Boulais à Bouchamples-Craon (100 m sur 118, soit 1,2 ha: Leroux et al., 1999, p. 274). Il présente également un fossé de partition interne. Orientés est/ouest, les accès principaux se situent sur son grand axe. Il existe également un tel enclos aux Mazures à Niafles (90 m sur 130 , soit 1,17 ha : ibid., p. 268) et un autre à la Petite Rouairie, commune de La Selle-Craonnaise (146 m sur 86, soit plus de 1,25 ha : ibid., p. 291). Un dernier, le site du Chef Lieu à Pommerieu (ibid., p. 291), plus proche du Clos-Henry par la surface puisqu'il ne mesure que $70 \mathrm{~m}$ sur 90 (0,63 ha), appartient également à ce type; le fossé de partition interne n'y est pas exactement au centre de l'espace.

Mais ce type d'enclos n'est pas propre à cette partie des confins de la Bretagne et des Pays de la Loire; il suffit de citer la fouille récente du site de l'Echasserie à Chemillé (Maine-et-Loire), repéré sur le tracé de l'autoroute A 87 et évalué lors de l'aménagement de la "route des Mauges " (Pinard, 2003, p. 32-33). La surface de cet établissement occupe au moins $6000 \mathrm{~m}^{2}$, une partie du site se trouvant hors emprise de l'étude.

Deux hypothèses sont à discuter ici : la présence d'une entrée au milieu du côté sud-oriental de l'enclos et un chemin de desserte.

Les enceintes rectangulaires citées plus haut montrent toutes une interruption du fossé au centre d'un de leurs petits côtés (Leroux et al., 1999, p. 59-60). Cette disposition, qui se répète au niveau du fossé de partition interne, pourrait également se retrouver au Clos-Henry : on y note, d'une part, une lacune au centre du fossé qui divise l'espace en deux parties et, d'autre part, les rares sondages ouverts dans le fossé sud-est de l'enceinte juste avant sa destruction ont livré un mobilier (tessons et éléments de terre cuite) plus abondant que partout ailleurs dans l'enclos. La présence de cette zone de concentration pourrait être liée à la proximité de l'entrée. Avec un fossé qui vient doubler ce côté de l'enclos, il semble également possible de restituer un chemin qui viendrait du nord-est et qui desservirait cet accès (fig. 16).

\section{Conclusion générale}

La fouille du site du Clos-Henry a permis pour la première fois en Mayenne, et plus particulièrement dans le sud-ouest d'un département extrêmement riche en sites enclos, d'étudier un type d'établissement rural repéré à de multiples reprises en photographie aérienne. Le mobilier, peu abondant mais très homogène, montre qu'il s'agit ici d'une création de la seconde moitié du II siècle avant J.-C., abandonnée vers le milieu du siècle suivant. La durée d'oc- 


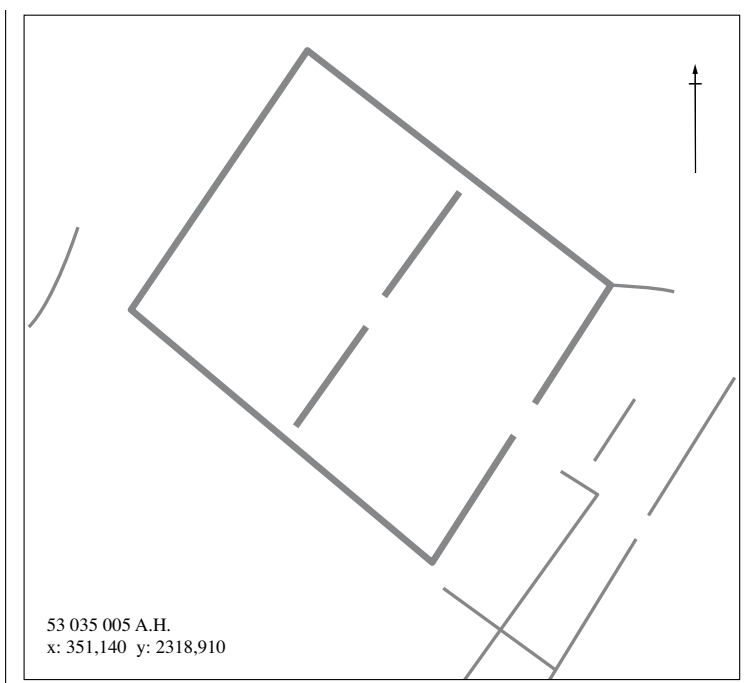

180. BOUCHAMPS-LES-CRAON (53) - Les Boulais.

Enclos rectangulaire possédant une partition interne. Son entrée, au sud-est, est aménagée par un dispositif d'antennes et de chicanes.

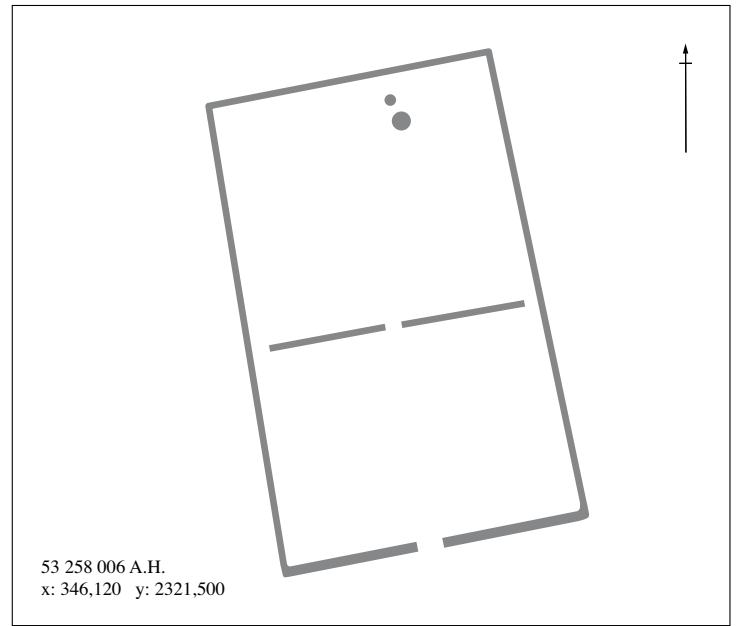

172. LA SELLE-CRAONNAISE (53) - La Petite Rouairie.

Grand enclos rectangulaire possédant une partition interne et une entrée au sud. On note la présence de deux fosses circulaires sur son coté nord.

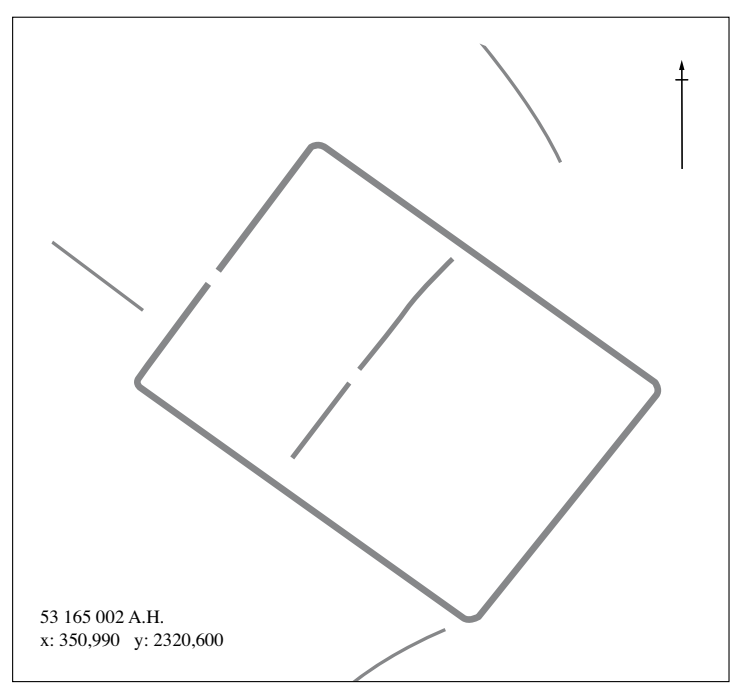

178. NIAFLES (53) - Les Mazures.

Enclos rectangulaire possédant une partition interne et une entrée face au nord-ouest. On note la présence de probables limites agraires associées.

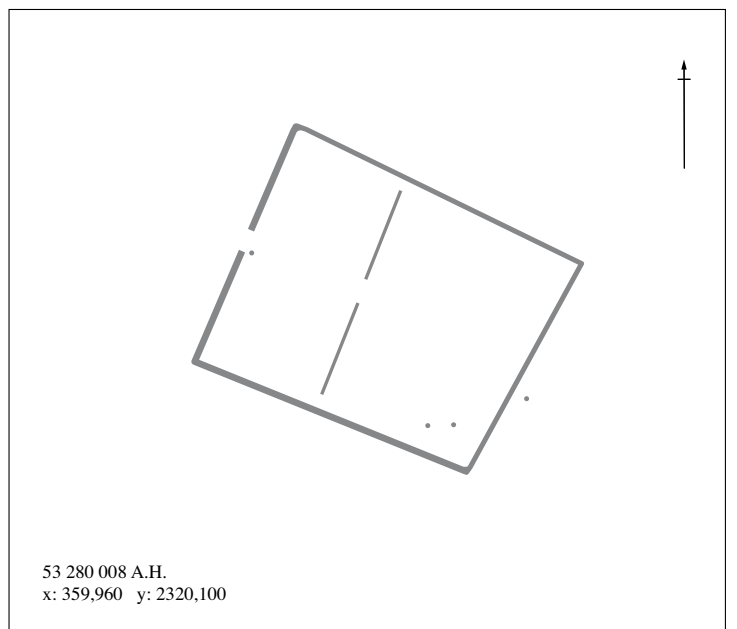

201. POMMERIEUX (53) - Le Chef Lieu.

Enclos rectangulaire possédant une partition interne. Une entrée aménagée est visible au nord-ouest. On note la présence de fosses dans son angle sud-ouest..

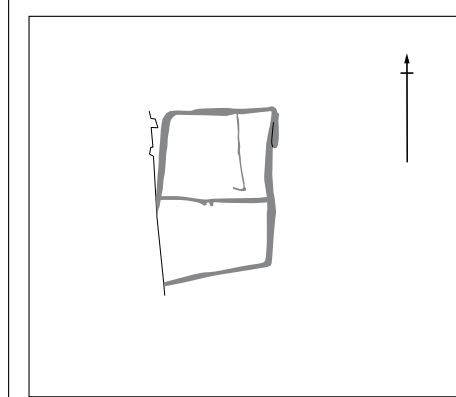

VIVOIN (72), La Pièce de Bildoux

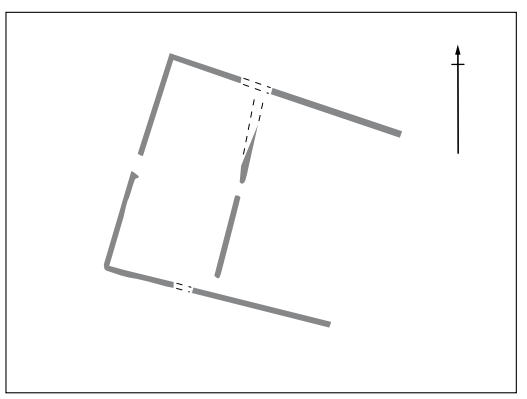

CHEMILLE (49), L'Echasserie.

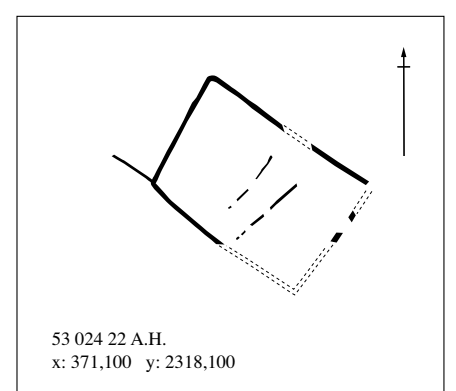

CHATEAU-GONTIER / BAZOUGES (53) Le Clos Henry.

Sites de références : clichés et redressement : G. LEROUX / D.A.O : S. JEAN.

Figure 15: Quelques exemples d'enclos rectangulaires à partition interne de la région.

Figure 15: Some regional examples of rectangular enclosures with internal partition. 
Figure 16 : La ferme du Clos-Henry, proposition de plan restitué.

Figure 16: Proposed reconstructed plan for Clos-Henry farmstead.

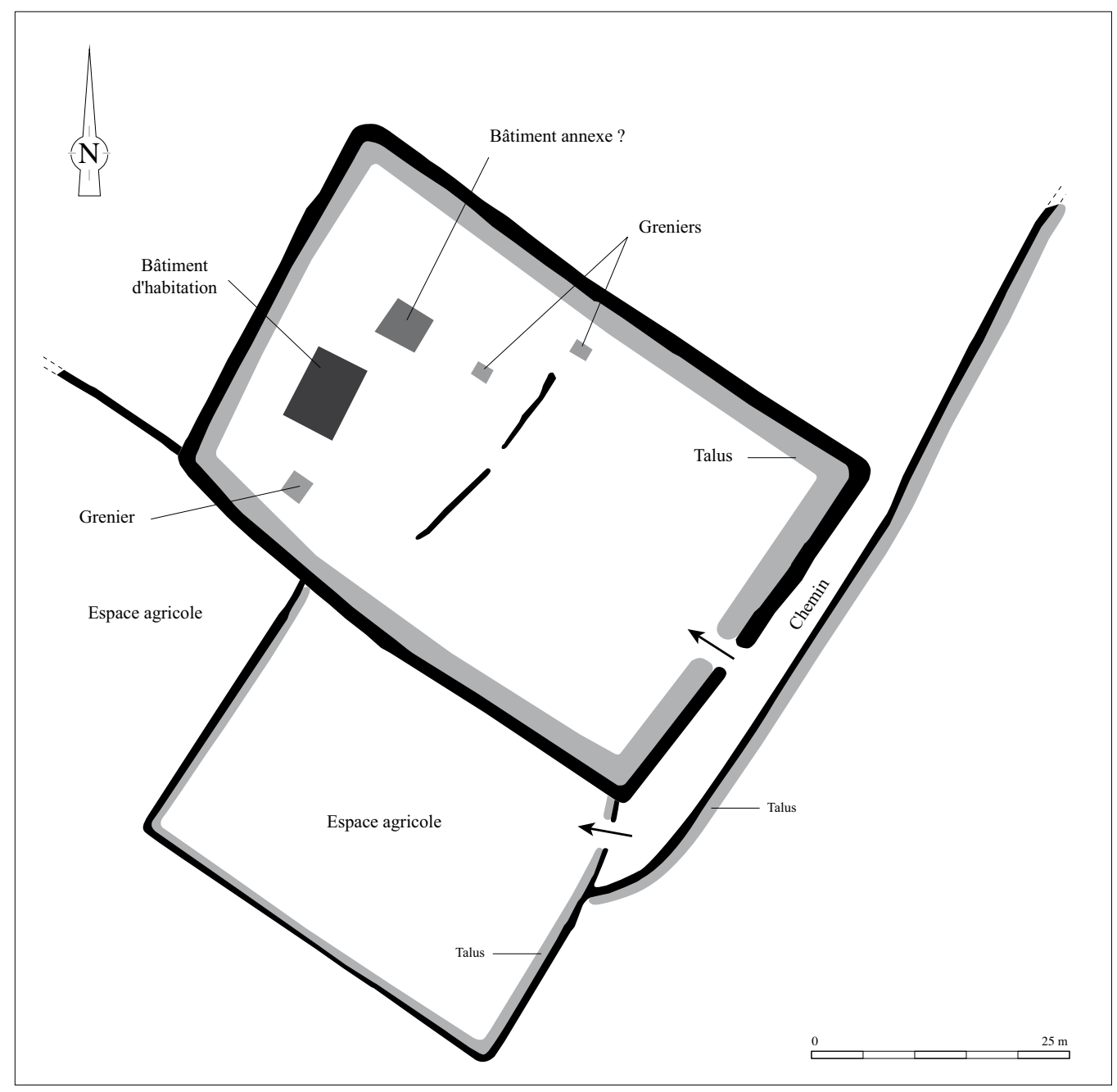

cupation du site apparaît assez courte et on y dénombre peu de structures mais un nombre de bâtiments proportionnellement très élevé, où seule l'habitation présente des traces de restauration. Durant une ou deux générations, cet enclos rectangulaire de $2400 \mathrm{~m}^{2}$ semble partagé entre un secteur arrière réservé aux activités domestiques et de stockage et une partie avant qui paraît moins densément construite. En raison de la destruction de cette partie du site avant notre intervention, nous manquons cependant d'éléments précis pour en connaître exactement l'occupation. S'agit-il de parcs à bestiaux ou d'un secteur à vocation maraîchère? Rien ne permet de trancher. Un espace agricole pourrait également avoir existé au sud de l'enclos habité.

Si ces éléments sont habituels pour une ferme de cette période, ce qui l'est moins dans la région, c'est sa très faible emprise. Un seul site plus petit a été relevé, celui de la Pièce de Bildoux à Vivoin (Sarthe) avec seulement $1500 \mathrm{~m}^{2}$ (Maguer et al., 2003, p. 217). Les établissements fouillés et ceux repérés du ciel sont généralement beaucoup plus vastes et peuvent atteindre par exemple $28000 \mathrm{~m}^{2}$ aux Genâts en
Vendée (Nilesse, 2003, p. 278). Les surfaces encloses, extrêmement variables d'un site à l'autre, sont un des éléments qui permettent d'en hiérarchiser les occupants. À ces amplitudes d'emprises, viennent s'ajouter les gabarits de fossés plus ou moins larges (Ferdière et al., 2006, p. 26), mais aussi la qualité, le type (monnaies, armement, parures) et l'origine du mobilier découvert, notamment les quantités de céramiques d'importation comme les amphores (Malrain et al., 2002, p. 191-141). À la lumière de ces éléments, les occupants de la ferme du Clos-Henry apparaissent comme de bien modestes paysans. À travers cette découverte, c'est une fois encore l'importance de l'occupation humaine de cette région du sud-ouest du département de la Mayenne qui est mise en lumière, mais c'est aussi, à travers la profusion de sites enclos repérés dans cette région de confins, la diversité de ces unités agricoles qui transparaît. Non loin des exploitations aurifères qu'étaient alors les "Miaules " (Meuret, 1993, p. 228-249), ce tissu d'établissements fossoyés a contribué également à mettre en valeur ce terroir de l'est-armoricain. 


\section{Bibliographie}

Angot, A., 1900-1910 - Dictionnaire historique, topographique de la Mayenne, Laval, Goupil, 4 tomes.

Boissel, R. et Naveau, J., 1980 - Les fouilles du terrain de sport de Jublains (Mayenne) - 1972-1979, La Mayenne : archéologie, histoire, 2, p. 3-13, $21 \mathrm{pl}$.

Bouvet, J.-P., 1997 - Les céramiques de l'âge du Fer, in Naveau, J., (dir.) - Recherches sur Jublains (Mayenne) et sur la cité des Diablintes, Rennes, éd. RAO, coll. "Documents archéologiques de l'Ouest ", p. 222-240.

Bouvet, J.-P., Brodeur, J., Chevet, P., Mortreau, M. et Siraudeau, J., 2003 - Un oppidum au château d'Angers (Maine-et-Loire), Revue archéologique de l'Ouest, suppl. 10 [Mandy, B et de Saulce, A. (dir.), Les marges de l'Armorique à l'âge du Fer. Archéologie et Histoire; culture matérielle et sources écrites (actes du XXIII colloque de l'AFEAF, Nantes 1999)], p. 173-187.

Cassen, S., Audren, C., Hinguant, S., Lannuzel, G. et Marchand, G., 1998 - L'habitat Villeneuve-Saint-Germain du Haut Mée (Saint-Etienne en Coglès, Ille-et-Vilaine), Bulletin de la Société préhistorique française, 95, p. 41-45.

Chancerel, A., Ghesquière, E., Lepaumier, H., Forfait, N., et LeCLERC, G., 1995 - Nouvelles implantations du VilleneuveSaint-Germains en Basse-Normandie, Revue archéologique de l'Ouest, suppl. 7 [Billard, C (dir.) - actes du XX $\mathrm{XX}^{\mathrm{e}}$ colloque interrégional sur le Néolithique (Evreux 1993)], p. 43-56.

CoRnu, S., 1997 - Céramiques de la moyenne vallée de la Loire à la fin de l'âge du Fer (thèse de doctorat), Université de Nantes, 2 vol.

DaIre, M.-Y, 1991 - Les céramiques gauloises de l'Homme-Mort, in Leroux, G. (dir.), Un établissement gaulois de Haute-Bretagne. La fouille du site de l'Homme-Mort en Saint-Pierre-de-Plesguen (Ille-et-Vilaine), Saint-Malo, éd. CeRAA, p. 33-73.

DAIRE, M.-Y., 1992 - Les céramiques armoricaines de la fin de l'âge $d u$ Fer, Rennes, Travaux du Laboratoire d'Anthropologie, (39), $316 \mathrm{p}$.

Ferdière, A., Malrain, F., Matterne, V., Méniel, P. et NissenJaubert, A., 2006 - Histoire de l'agriculture en Gaule de 500 av. J.-C. à 1000 ap. J.-C., Paris, éd. Errance, 231p.

GransAR, F., 2000 - Le stockage alimentaire sur les établissements ruraux de l'âge du Fer en France septentrionale : complémentarité des structures et tendances évolutives, in MARION, S. et Blancquaert, G. - Les installations agricoles de l'àge du Fer en France septentrionale, Paris, Ecole normale supérieure, p. $277-$ 297.

Gruet, M., 1986 - Le sol d'habitat néolithique Augy-SaintePallaye de la Bajoulière, (Maine-et-Loire), Revue archéologique de l'Ouest, Suppl. 1 (actes du $\mathrm{X}^{\mathrm{e}}$ colloque Interrégional sur le Néolithique, Caen 1983), p. 137-141.

Hinguant, S., Le Goff, E., Jean, S. et Marguerie, D., 1997 - Le site gaulois de Bellevue à Augan. Un établissement rural en limite de deux influences armoricaines, Revue archéologique de l'Ouest, 14, p. 57-80.

Hinguant, S. et Le Goff, E., 1998 - Un site de l'âge du Fer stratifié en milieu rural : l'établissement de Kéralio à Pont-Labbé (Finistère), Revue archéologique de l'Ouest, 15, p. 59-114.

LAmbert, C. et Rioufreyt, J., 1976 - Des enceintes protohistoriques aux sanctuaires gallo-romains, Bulletin de la Commission historique et archéologique de la Mayenne, 41-42, p. 3-60.

LANGLOIS, J.-Y., 2001 - Château-Gontier "Rocade nord " (rapport de diagnostic archéologique), AFAN/Service régional de l'Archéologie des Pays de la Loire, $27 \mathrm{p}$

Legros, S., 2006 - Les prieurés de Château-Gontier et l'établissement d'une seigneurie châtelaine dans le comté d'Anjou (fin $\mathrm{du} \mathrm{x}^{\mathrm{e}}$ siècle-fin du XI ${ }^{\mathrm{e}}$ siècle), Annales de Bretagne et des Pays de l'Ouest, 113, p. 33-59.

Le Goff, E., 2003 - "Armorique et Bretagne " : réflexion sur l'assimilation de ces concepts pour la fin de l'âge du Fer, Revue archéologique de l'Ouest, suppl. 10 [MANDy, B et DE SAulce, A. (dir.), Les marges de l'Armorique à l'âge du Fer. Archéologie et Histoire; culture matérielle et sources écrites (actes du XXIII ${ }^{\circ}$ colloque de l'AFEAF, Nantes 1999)], p. 103-117.

Leroux, G., Gautier, M., Meuret, J.-C. et NaAs, P., 1999 Enclos Gaulois et gallo-romains en Armorique, de la prospection aérienne à la fouille entre Blavet et Mayenne. Rennes, éd. RAO coll. "Documents archéologiques de l'Ouest ", 335 p.

Maguer, P., Chérel, A.-F. et Auxiette, G., 2003 - Nouvelles données sur les habitats de l'Âge du Fer en Sarthe (commune de Vivion, autoroute A28), Revue archéologique de l'Ouest, suppl. 10 [Mandy, B et DE Saulce, A. (dir.), Les marges de l'Armorique à l'âge du Fer. Archéologie et Histoire; culture matérielle et sources écrites (actes du XXIII ${ }^{\mathrm{e}}$ colloque de l'AFEAF, Nantes 1999)], p. 213-234.

Malrain, F., Matterne, V. et Méniel, P., 2002 - Les paysans gaulois (III ${ }^{\mathrm{e}}$ siècle-52 avant J.-C.), Paris, éd. Errance, 235 p.

MénEz, Y., 1996 - Une ferme de l'Armorique gauloise, Le Boisanne à Plouer-sur-Rance (Côtes-d'Armor), Paris, Maison des Sciences de l'Homme (coll. Documents d'Archéologie française), 267 p.

Meuret, J.-C., 1991 - Forêt de La Guerche, Ligne Anne, Rannée (Ille-et-Vilaine) (rapport intermédiaire de fouille programmée bi-annuelle), Rennes, SRA Bretagne.

Meuret, J.-C., 1992 - Forêt de La Guerche, Ligne Anne, Rannée (Ille-et-Vilaine) (rapport de fin de fouille programmée biannuelle), Rennes, SRA de Bretagne.

Meuret, J.-C., 1993 - Peuplement, pouvoir et paysage sur la marche Anjou-Bretagne (des origines au Moyen Âge). Société d'Archéologie et d'Histoire de la Mayenne, suppl. 4, 656 p.

Meuret, J.-C., 1997 - Peuplement ancien de la haute vallée de l'Oudon (Mayenne), La Glanerie, Athée. (rapport de fouille programmée annuelle), Nantes, SRA des Pays de la Loire, 87 p.

Meuret, J.-C., 2000 - Habitats enclos de Haute-Armorique : de l'avion à la fouille, in Marion, S. et Blancquaert, G. (dir.), 
Les installations agricoles de l'âge du Fer en France septentrionale. Paris, Ecole Normale Supérieure, p. 75-102.

Meuret, J.-C., 2003 - De l'avion au terrain : données et problèmes de la recherche sur les enclos aux marges de la Bretagne. Revue archéologique de l'Ouest, suppl. 10 [MANDY, B et DE SAulce, A. (dir.), Les marges de l'Armorique à l'âge du Fer. Archéologie et Histoire; culture matérielle et sources écrites (actes du XXIII' colloque de l'AFEAF)], p. 23-36.

Meuret, J.-C., 2006 - La Montagne, Visseiche (Ille-et-Vilaine) (rapport de fouille programmée annuelle), Rennes, SRA de Bretagne, 61 p. et 127 fig.

Naveau, J., 1976 - L'oppidum de Moulay (travaux de l'année 1975). Bulletin de la Commission historique et archéologique de la Mayenne, 41-42, p. 61-98.

Naveau, J., 1977 - Fouille d'un fossé de l'âge du Fer dans la carrière d'Ecorcé à Entrammes, Bulletin de la Commission historique et archéologique de la Mayenne, 46, p. 3-23.

Naveau, J., 1992 - Carte Archéologique de la Gaule - La Mayenne. Paris, Académie des Inscriptions et Belles lettres/ministère de l'Éducation Nationale et de la Culture, 176 p.

Naveau, 1997 - Recherches sur Jublains (Mayenne) et sur la cité des Diablintes, Rennes, éd. RAO, coll. «Document archéologiques de l'Ouest ", Rennes, 352 p., 3 pl.

NiLESSE, O., 2003 - Les établissements ruraux gaulois de la plaine de Luçon (Vendée), Revue Archéologique de l'Est, 20 suppl.
(Habitat, Mobiliers et groupes régionaux à l'Âge du Fer, actes du XX $\mathrm{XX}^{\mathrm{e}}$ colloque de l'AFEAF, Colmar-Mittelwir, 1996), p. $275-287$.

Pinard, F., 2003 - Chemillé "L'Echasserie ", Bilan scientifique des Pays de La Loire pour l'année 2000, DRAC des Pays de La Loire, p. 32-33.

VACHer, S. et Bernard, V., 2003 - Un site en zone inondable : Le grand Aunay à Yvré-l'Evêque, Revue archéologique de l'Ouest, suppl. 10 [MAndy, B et de Saulce, A. (dir.), Les marges de l'Armorique à l'âge du Fer. Archéologie et Histoire; culture matérielle et sources écrites (actes du XXIII ${ }^{\mathrm{e}}$ colloque de l'AFEAF)], p. 289-212.

VAlaIs, A., 2001 a - Château-Gontier / Bazouges "Montaigu » (rapport de diagostic), Nantes, SRA des Pays de La Loire, 9p.

ValaIs, A., 2001 b - "Vauvert " à Château-Gontier / Bazouges. (DFS de fouilles archéologiques), Nantes, SRA des Pays de La Loire, $41 \mathrm{p}$.

Valais, A., 2001c - Le site du Clos-Henry (Château-Gontier / Bazouges), (DFS d'évaluation), Nantes, SRA des Pays de la Loire.

Valais, A., 2004 - L'agglomération antique d'Entrammes enfin découverte, La Mayenne Archéologie Histoire, n 27, p. 12-13.

Valais, A., 2008 - Premier bilan des découvertes sur le tracé de la rocade de Mayenne, La Mayenne Archéologie Histoire, $\mathrm{n}^{\circ} 30$, p. 200-217. 
